\title{
Simulations and Analytic Calculations of Bubble Growth during Hydrogen Reionization
}

\section{Citation}

Zahn, Oliver, Adam Lidz, Matthew McQuinn, Suvendra Dutta, Lars Hernquist, Matias Zaldarriaga, and Steven R. Furlanetto. 2007. "Simulations and Analytic Calculations of Bubble Growth during Hydrogen Reionization." The Astrophysical Journal 654 (1): 12-26. https:// doi.org/10.1086/509597.

\section{Permanent link}

http://nrs.harvard.edu/urn-3:HUL.InstRepos:41381780

\section{Terms of Use}

This article was downloaded from Harvard University's DASH repository, and is made available under the terms and conditions applicable to Other Posted Material, as set forth at http:// nrs.harvard.edu/urn-3:HUL.InstRepos:dash.current.terms-of-use\#LAA

\section{Share Your Story}

The Harvard community has made this article openly available. Please share how this access benefits you. Submit a story. 
November 5, 2018. Submitted to ApJ.

Preprint typeset using $\mathrm{LAT}_{\mathrm{E}} \mathrm{X}$ style emulateapj v. 11/10/09

\title{
SIMULATIONS AND ANALYTIC CALCULATIONS OF BUBBLE GROWTH DURING HYDROGEN REIONIZATION
}

\author{
Oliver Zahn ${ }^{1}$, Adam Lidz ${ }^{1}$, Matthew McQuinn ${ }^{1}$, Suvendra Dutta $^{1}$, Lars Hernquist ${ }^{1}$, Matias Zaldarriaga $^{1,2}$, \\ Steven R. Furlanetto ${ }^{3}$ \\ November 5, 2018. Submitted to ApJ.
}

\begin{abstract}
We present results from a large volume simulation of Hydrogen reionization. We combine 3d radiative transfer calculations and an N-body simulation, describing structure formation in the intergalactic medium (IGM), to detail the growth of HII regions around high redshift galaxies. Our N-body simulation tracks $1024^{3}$ dark matter particles, in a cubical box of co-moving side length $L_{\text {box }}=65.6$ $\mathrm{Mpc} h^{-1}$. This large volume allows us to accurately characterize the size distribution of HII regions throughout most of the reionization process. At the same time, our simulation resolves many of the small galaxies likely responsible for reionization. It confirms a picture anticipated by analytic models: HII regions grow collectively around highly-clustered sources, and have a well-defined characteristic size, which evolves from a sub-Mpc scale at the beginning of reionization to $R \gtrsim 10$ co-moving Mpc towards the end. We show that in order to obtain this qualitative picture, source resolution must not be sacrificed at too great a level. We present a detailed statistical description of our results, and compare them with a numerical hybrid scheme based on the analytic model by Furlanetto, Zaldarriaga, and Hernquist. This model associates HII regions with large-scale overdensities and is based on the excursion set formalism. We find that the analytic calculation reproduces the size distribution of HII regions, the power spectrum of the ionization field, and the $21 \mathrm{~cm}$ power spectrum of the full radiative transfer simulation remarkably well. The ionization field from the radiative transfer simulation, however, has more small scale structure than the analytic calculation, owing to Poisson scatter in the simulated abundance of galaxies on small scales. We propose and validate a simple scheme to incorporate this scatter into our calculations. Our results suggest that analytic calculations are sufficiently accurate to aid in predicting and interpreting the results of future $21 \mathrm{~cm}$ surveys. In particular, our fast numerical scheme is useful for forecasting constraints from future $21 \mathrm{~cm}$ surveys, and in constructing mock surveys to test data analysis procedures.
\end{abstract}

Subject headings: cosmology: theory - intergalactic medium - large scale structure of universe

\section{INTRODUCTION}

The epoch of reionization (EOR) is a pivotal stage in the process of cosmological structure formation, marking the birth of the first luminous objects, a key landmark as the universe transforms from the relatively smooth state probed by the cosmic microwave background (CMB), to its present day complexity. Our current observational constraints on reionization come from Ly $\alpha$ forest absorption spectra towards high redshift quasars and constraints on the evolution of the ionizing background (e.g. Fan et al. 2005), from measurements of the high redshift galaxy luminosity function from narrow-band Ly $\alpha$ emission searches (Malhotra \& Rhoads 2005), and from measurements of the large scale CMB E-mode polarization (Page et al. 2006; Spergel et al. 2006). The claimed size of HII regions surrounding individual quasars has also been used to infer limits on the neutral fraction (Mesinger et al. 2004, Wyithe et al. 2004). While valuable, each of these observational probes has its limitations, and some of the current constraints are relatively

\footnotetext{
ozahn@cfa.harvard.edu,alidz@cfa.harvard.edu

${ }^{1}$ Harvard-Smithsonian Center for Astrophysics, 60 Garden Street, Cambridge, MA 02138

2 Jefferson Laboratory of Physics; Harvard University; Cambridge, MA 02138

3 Yale Center for Astronomy and Astrophysics, Yale University, 260 Whitney Avenue, New Haven, CT 06520-8121
}

meager. Quasar absorption spectra are limited in part by the high Ly $\alpha$ absorption cross section: by $z \sim 6$, even a highly ionized IGM completely absorbs quasar flux in the Ly $\alpha$ forest. The constraints from narrowband Ly $\alpha$ searches are subtle to interpret (e.g. Furlanetto et al. 2006b), and restricted to narrow redshift windows around $z=5.7$ and $z=6.5$, where Ly $\alpha$ falls in the observed optical band, and avoids contamination from bright sky lines (e.g. Rhoads et al. 2003). The CMB polarization measurements constrain only an integral over the ionization history, and are potentially sensitive to foreground contamination (Kogut et al. 2003, Page et al. 2006).

The study of reionization may be revolutionized by future experiments aimed at detecting $21 \mathrm{~cm}$ emission from the high redshift IGM. These experiments should provide three-dimensional information regarding the distribution of high redshift neutral hydrogen, constraining the topology of reionization, and its redshift evolution (e.g. Madau et al. 1996, Zaldarriaga et al. 2004). Several low frequency radio telescopes are presently ramping up to detect this signal: the Mileura Wide Field Array (MWA) (Bowman et al. 2006) 4 the PrimeavAl Structure Telescope (PAST) (Pen et aI. 2004), and the

\footnotetext{
${ }^{4}$ http://web.haystack.mit.edu/arrays/MWA/
} 
Low Frequency Array (LOFAR) 5, while another second generation experiment, the Square Kilometer Array $(\mathrm{SKA})^{6}$ is in the planning stage. These measurements will be dominated by foreground contamination, but in contrast to the IGM signal, the foregrounds are expected to be smooth in frequency, facilitating their removal (Zaldarriaga et al. 2004). The $21 \mathrm{~cm}$ data will be supplemented by further quasar absorption spectra (including clues from metal absorption lines: Oh 2002, Becker et al. 2005), high redshift gamma ray bursts (e.g. Barkana \& Loeb 2004a; Totani et al. 2005), high redshift galaxy surveys (e.g. Kneib et al. 2004), and small-scale CMB measurements (Santos et al. 2003; Zahn et al. 2005, McQuinn et al. 2005), providing a wealth of observational data on the process of reionization.

Detailed theoretical modeling (see e.g. Gnedin 2000, Razoumov et al. 2002, Ciardi et al. 2003; Sokasian et al. 2002, Sokasian et al. 2001; Furlanetto et al. |2004b a; Kohler et al. 2005; Iliev et al. 2005 Mellema et al. 2006) is, however, required to constrain the topology of reionization from current and future observations. In particular, it is subtle to infer quantities like the volume filling factor and size distribution of ionized regions, the correlation between ionized regions and large-scale over-density (i.e., does reionization proceed outside-in or inside-out), and the nature of the ionizing sources, from observations.

Unfortunately, numerical modeling of reionization is challenging, requiring treatment of radiative transfer, preferably some treatment of gas dynamics, and a large dynamic range. A large dynamic range is required to resolve the small mass galaxies which may make up the sources and sinks of ionizing photons, while simultaneously sampling the distribution of HII regions, which may be as large as $R \sim 20$ comoving Mpc $h^{-1}$ towards the end of reionization (Furlanetto \& Oh 2005). For this reason, most calculations have been performed in prohibitively small simulation boxes, or been entirely analytic (e.g. Furlanetto et al. 2004a, hereafter FZH04), although there has been very recent progress towards large volume reionization simulations (Kohler et al. 2005: Iliev et al. 2005). Indeed a skeptic might posit that, given the difficulty of simulating reionization, we will observe the $21 \mathrm{~cm}$ signal before we can predict it.

In this paper, we push forward by running a large volume radiative transfer simulation. Our work represents progress on several fronts. First, we simulate reionization in a larger volume than most previous works (although see Kohler et al. 2005, Iliev et al. 2005), while maintaining high mass resolution. This allows us to reliably calculate the size distribution of HII regions as well as power spectra of ionization and $21 \mathrm{~cm}$ fields, impossible with previous small volume simulations. Second, we compare our results with analytic calculations based on FZH04. These models are now widely used, and while elegant and inspired by previous small volume reionization simulations (Sokasian et al. 2003, 2004), they remain untested. Our comparison also gauges the level of theoretical control in our modeling of reionization - i.e., how robust are our conclusions to the details of our modeling? One convincing way to dissuade the above-mentioned skeptic is to demonstrate that we can understand the gross features

\footnotetext{
${ }^{5}$ http://www.lofar.org

6 http://www.skatelescope.org/
}

of our radiative transfer simulations analytically. Additionally, if analytic models are sufficiently accurate then they are useful tools to forecast constraints from future experiments, and to construct mock surveys, providing important tests of data analysis procedures. This is important given our ignorance of the nature of the ionizing sources: we would like to cover a large parameter space in the source properties, prohibitive with time-consuming radiative transfer simulations. Furthermore, future surveys will span volumes of several cubic Giga-parsecs, a challenging task for detailed simulations.

We emphasize that our present work is only a first step towards more realistic simulations of Hydrogen reionization. As we describe subsequently, our radiative transfer simulations miss potentially important aspects of the physics of reionization. Specifically, we include only a crude prescription for the sources of ionizing photons, our coarse resolution underestimates the importance of recombinations - especially if mini-halos are present during reionization (Haiman et al. 2000, Barkana \& Loeb 2002 Shapiro et al. 2004) - and misses small galaxies that may contribute ionizing photons, and we ignore feedback effects entirely. We intend to model some of these effects in the near future (McQuinn et al. 2006)).

Our work has overlap with the recent simulation and analysis of Iliev et al. (2005). In comparison to these authors, reionization finishes significantly later in our simulation, near $z \sim 6.5$, as compared to $z \sim 12$, a consequence of our more conservative prescription for the ionizing sources. Moreover, our main present emphasis is in comparing our radiative transfer simulation results with 'hybrid simulations' based on analytic models.

The layout of this paper is as follows. In $\$ 2$ we describe our N-body simulation, source prescription and radiative transfer calculation. In $\$ 3$ we describe our 'analytic model simulation', which is more precisely an implementation of a model based on FZH04 into the cosmological realization used for the radiative transfer simulation. We will sometimes refer to this scheme losely as an 'analytic calculation' although the implementation of the model is entirely numerical. In 84 we present a detailed statistical description of our radiative transfer and analytic results. We describe a numerical scheme that incorporates the stochasticity of the source distribution into our analytic calculations in $\$ 5$. We also show that if extremely bright and rare sources reionize the IGM, bubble growth is less collective than in our fiducial model.

In $\$ 6$ we compare radiative transfer and analytic model predictions for the $21 \mathrm{~cm}$ signal. We conclude in $\$ 7$. mentioning future research directions and emphasizing possible improvements to our simulations.

Throughout we assume a flat, $\Lambda \mathrm{CDM}$ cosmology parameterized by: $\Omega_{m}=0.3, \Omega_{\Lambda}=0.7, \Omega_{b}=0.04$, $H_{0}=100 h \mathrm{~km} / \mathrm{s} / \mathrm{Mpc}$ with $h=0.7$, and a scale-invariant primordial power spectrum with $n=1$, normalized to $\sigma_{8}(z=0)=0.97$

7 This value for $\sigma_{8}$ is slightly different than the value preferred by the WMAP satellite alone of $0.76 \pm 0.05$ (Spergel et al. 2006 ) When combined with other datasets, such as the Lyman- $\alpha$ torest (Lewis 2006. Seljak et al. 2006), and weak lensing (see e.g. section 4.1.7 and Table 6 of Spergel et al. 2006), a higher value of $\sigma_{8}$ can be found. Furthermore, changes in the fluctuation amplitude within the present experimental boundaries can be incorporated into our analysis by adjusting slightly the ionization efficiency pa- 


\section{SIMULATIONS}

We begin by running a large N-body simulation to locate dark matter halos, and produce a cosmological density field. Next, we populate the dark matter halos with ionizing sources, using a simple prescription to connect mass and light $(2.2$. In a subsequent post-processing step, we perform a radiative transfer calculation, casting rays of ionizing photons from our sources through the cosmological density field (\$2.3). We make two approximations with this approach. First, we assume that the gas distribution perfectly traces the dark matter distribution, as characterized by our N-body simulation. Second, we neglect the interplay between gas dynamics and radiation transport - i.e, in reality, structure formation responds to the passage of ionization fronts, and gas motions in turn influence the propagation of the fronts. These effects are essential in calculating the detailed small-scale behavior of ionization fronts, as fronts slow down upon impacting dense clumps (Shapiro et al. 2004), but are less important for our goal of capturing the large-scale size distribution of HII regions.

\subsection{N-body simulations}

As noted in the introduction, we require a cosmological simulation with a large dynamic range, in order to adequately sample the distribution of HII regions, while simultaneously resolving small galaxies. Ideally, we would resolve halos with virial temperatures of $T_{\text {vir }} \gtrsim 10^{4} \mathrm{~K}$ - corresponding to a dark matter halo mass of $M_{\mathrm{dm}} \sim 10^{8} M_{\odot}$ at $z \sim 6$ - above which atomic line cooling is efficient. In halos more massive than this, gas can cool, condense to form stars, and produce ionizing photons. This 'cooling mass' therefore represents a plausible guess as to the minimum host halo mass for ionizing sources. If molecular Hydrogen cooling is efficient despite radiative feedback, however, even smaller mass halos should host sources (Haiman et al. 1997). Presently, we ignore this possibility. Additionally, high resolution is required to capture the clumpiness of the IGM, and properly account for recombinations during reionization. On the other hand, HII regions may be larger than $R \geq 20$ $\mathrm{Mpc} h^{-1}$ at the end of reionization (Furlanetto \& Oh 2005), necessitating a large volume simulation. Unfortunately, to resolve a $10^{8} M_{\odot}$ halo with 32 particles, in a simulation box of side-length $L=100 \mathrm{Mpc} h^{-1}$, for example, requires a prohibitively large number of particles, $N_{p} \sim 3360^{3}$ !

Our present N-body simulation is meant to represent a compromise between these competing requirements of large volume, and high mass resolution. Specifically, our N-body simulation follows $1024^{3}$ dark matter particles in a box of side-length, $L=65.6 \mathrm{Mpc} h^{-1}$, using an enhanced version of the TreePM code, Gadget-2 (Springel 2005). We run the simulation assuming the flat LCDM cosmology specified in the introduction, with initial conditions generated using the Eisenstein \& Hu (1999) transfer function.

Dark matter halos are identified from simulation snapshots, using a friends-of-friends algorithm (e.g., Davis et al. 1985). Specifically, particles are grouped into halos

rameter. This does not qualitatively affect our results, as we confirmed within the analytic scenario.

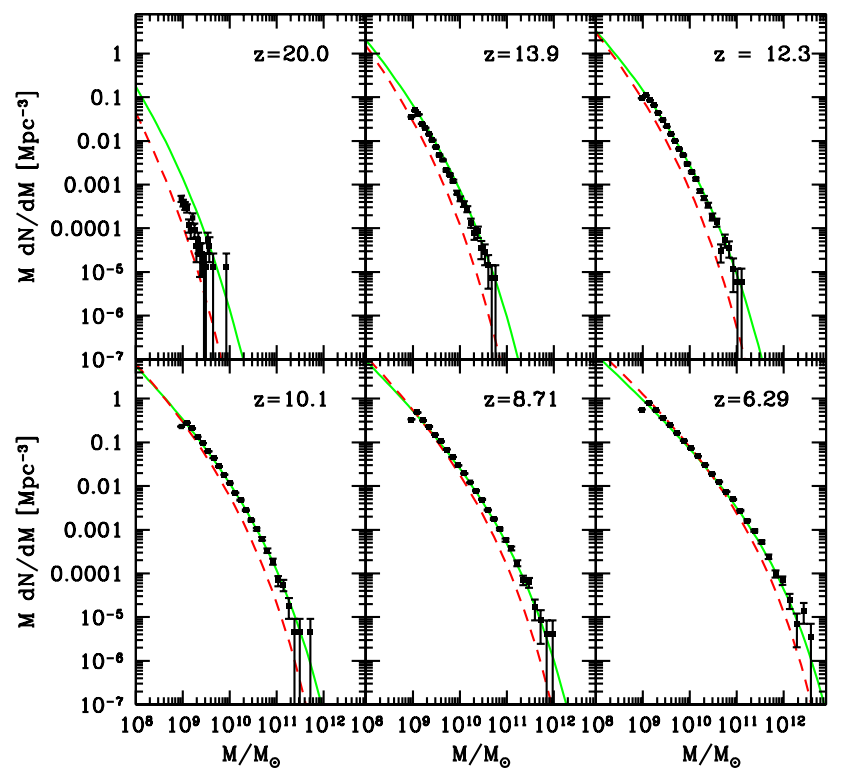

FIG. 1.- Halo mass function from our N-body simulation. The black points with (Poisson) error bars indicate the halo mass function from our simulation as a function of redshift. The green curve is the Sheth-Tormen fitting function for the halo mass function, while the red dashed line shows the Press-Schechter fitting function.

using a linking length of $b=0.2$ times the mean interparticle separation. Linked groups of greater than 32 particles are considered resolved, and to constitute dark matter halos. This corresponds to a minimum halo mass of $10^{9} M_{\odot}$, just an order of magnitude above the cooling mass.

The resulting mass function is shown in Figure 1, spanning a broad redshift range between $z \sim 6-20$. Barkana \& Loeb (2004b) showed that if a simulation is normalized to the cosmic mean density, the halo mass functions will be biased. According to Figure 3 of Barkana \& Loeb (2004b), the bias introduced in our calculations should only be of order $0.1 \%$. The halo mass function is sampled with large dynamic range, roughly three orders of magnitude near $z \sim 6$. The simulated mass function is always larger than predicted by the Press-Schechter formalism (Press \& Schechter 1974), but generally in good agreement with the Sheth-Tormen (Sheth \& Tormen 1999) fitting formula. At the highest redshifts sampled, however, our results fall in between the two fitting formula. This is in qualitative agreement with recent measurements from Reed et al. (2005), and Heitmann et al. (2006), although our mass function appears systematically higher than that of Iliev et al. (2005). The figure shows that the abundance of our lowest mass halos is systematically below theoretical expectations, likely a consequence of our limited mass resolution. As a conservative measure, we therefore place ionizing sources only in halos of mass larger than $M_{\min }=2 \times 10^{9} M_{\odot}$, corresponding to a $64-$ particle halo.

\subsection{Ionizing Sources}


Our next step is to connect mass with light - that is, we wish to populate the dark matter halos from our Nbody simulation with ionizing sources. In this paper, we will adopt a very crude prescription for our ionizing sources, leaving a more sophisticated prescription to future work. This will facilitate comparison with the analytic models (see \$3). Specifically, we populate each dark matter halo with a single source whose luminosity in Hydrogen ionizing photons is directly proportional to the host halo mass, $\dot{N}=c M_{\text {halo. Clearly the pa- }}$ rameter $c$ encodes a good deal of complicated physics, involving the efficiency of star formation, the efficiency of producing ionizing photons, the fraction of ionizing photons that escape from the host halo, etc. With this single simplifying assumption, the cumulative number of ionizing photons released by the sources, per hydrogen atom in the IGM, at time $t$ is $N_{\mathrm{ph}} / N_{H} \propto \int_{0}^{t} d t^{\prime} f_{\text {coll }}\left(t^{\prime}\right)$. Here $f_{\text {coll }}\left(t^{\prime}\right)$ is the fraction of mass in halos with mass $M \geq M_{\min }=2 \times 10^{9} M_{\odot}$. Using the Sheth \& Tormen (1999) mass function, which closely matches our simulation results (Figure 1), we find that $c=3.1 \times 10^{41}$ photons $/ \mathrm{sec} / M_{\odot}$ yields one photon per hydrogen atom at $z=6.5$. (See Figure 2 and associated text for a discussion). This choice of $c$ corresponds roughly, for example, to Pop II stars, forming with an efficiency of $f_{\star}=0.1$ from a Salpeter IMF, with a stellar lifetime of $\Delta t \sim 5 \times 10^{7} \mathrm{yrs}$, and a modest escape fraction of $f_{\text {esc }} \sim 0.01$ (Loeb et al. 2005). We adopt this conversion in all subsequent calculations.

\subsection{Radiative Transfer}

We next form a coarse density field for many snapshots, spaced in equal time intervals of $\Delta t=5 \times 10^{7}$ years and spanning a broad redshift range from $z \sim 6-16$, by gridding our dark matter particles onto a uniform, Cartesian grid with $256^{3}$ mesh points. Our sources (\$2.1 and $\$ 2.2$ are tabulated at the same time-sampling, and moved close to the center of their corresponding cell. Occasionally, several sources land in a single cell and our considered to be a sole, more luminous source. At $z \sim 6.5$, near our assumed completion of reionization, there are $\simeq 330,000$ ionizing sources in our simulation.

With the ionizing sources and cosmological density field in hand, we trace rays of ionizing photons through the simulation box using the adaptive ray-tracing scheme of Abel \& Wandelt (2002), and the code of Sokasian et al. (Sokasian et al. 2001; Sokasian et al. 2003). Further improvements to the code were made (McQuinn et al. 2006). We refer the reader to these papers for the details of the code used here, but give a brief summary. In short, the code assumes a sharp ionization front, and tracks the position of the front by casting rays and integrating over the ionization front jump condition (Abel et al. 1998). The jump condition amounts to tabulating the number of photoionizations and recombinations along a ray, halting the ray when its photon supply is exhausted. Each source is considered separately, although the order in which sources are processed is randomized at each timestep to avoid artifacts (Sokasian et al. 2003).

Behind the ionization front, each source hitting a given cell contributes a photoionization rate of $\Gamma_{\mathrm{HI}, \mathrm{s}}=$ $\bar{\sigma} \dot{N} /\left(4 \pi r_{s}^{2}\right)$, i.e. assuming optically thin conditions within the front. Here $r_{s}$ is the distance from the cell

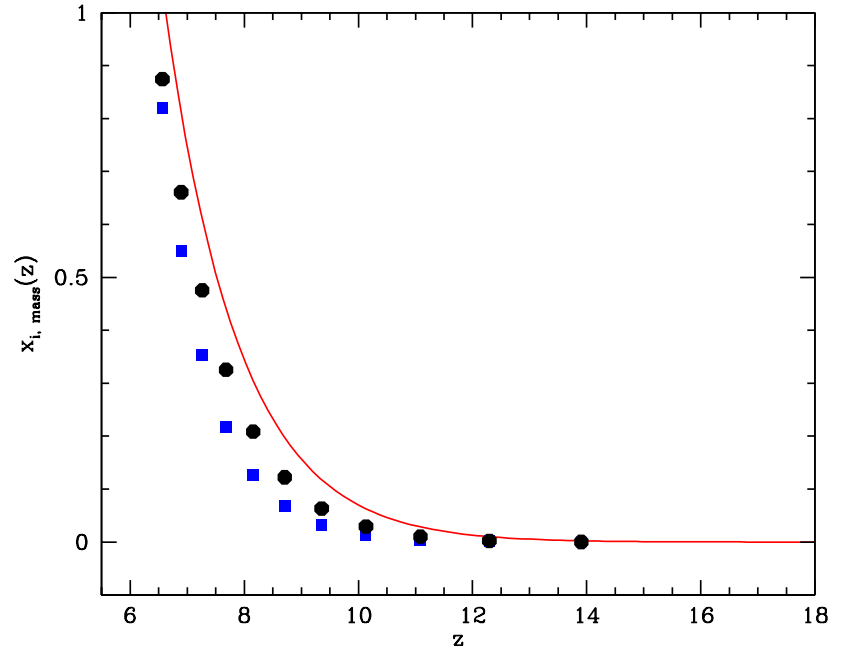

FIG. 2.- Ionization fraction as a function of redshift. The black circles show the mass-weighted ionization fraction from the simulation, while the blue squares show the volume-weighted ionization fraction. The red line is the cumulative number of ionizing photons per hydrogen atom expected for our ionizing sources. The close resemblance between the number of photons per atom and the measured ionization fractions owes to the poor resolution of our radiative transfer calculation, which underestimates the importance of recombinations.

in question to a source, $\dot{N}$ is the number of Hydrogen photons per second from a source, and $\bar{\sigma}$ is a frequencyaveraged cross section, computed here assuming each source has a spectrum $\propto \nu^{-4}$ (Sokasian et al. 2001). Within the front, ionization fractions are computed assuming ionization equilibrium and a uniform temperature of $T=10^{4} \mathrm{~K}$, and neglecting sub-grid clumping. We follow the approach of (Sokasian et al. 2001) in using case $\mathrm{B}$ recombination rates when casting rays through the grid, while using case A coefficients to compute the ionization fraction in previously ionized cells. Helium is assumed to be at most singly-ionized by our soft sources, and we assume that the HeII front precisely tracks the HII front. Similarly, inside the front we assume that the ionized Helium (HeII) fraction traces the HII fraction (Sokasian et al.2003). Note that all of these assumptions impact mainly the detailed ionization fractions within the front, and are less important for tracking the overall size distribution of HII regions. In contrast to Sokasian et al. (2001); Sokasian et al. (2003), we do not include a diffuse background radiation field, simply allowing rays to wrap around the periodic box.

Our assumption of a sharp ionizing front is justified given the short mean free path of Hydrogen ionizing photons in the pre-reionization IGM. Mellema et al. (2005) present explicit comparisons between ionization front tracking' and more detailed calculations that self consistently solve for the optical depth, ionization fraction, and temperature. At least in the case of a single source (their Figure 16), ionization front tracking reproduces very closely the results of more detailed calculations, further justifying our approach.

In Figure 2 we plot the redshift evolution of the ionization fraction in our simulation. The black circles show 
the mass-weighted ionization fraction, while the blue squares show the volume-weighted ionization fraction. The mass-weighted ionization fraction is somewhat larger than the volume-weighted ionization fraction. This is because the ionizing sources in our simulation are highly biased, and ionize their overdense environs before breakingfree to ionize neighboring voids (e.g. Sokasian et al. 2003 Iliev et al. 2005). The reionization process takes a fairly significant stretch of cosmic time, with the mass-weighted ionization fraction at the level of $x_{\mathrm{i}, \mathrm{m}} \sim 0.1$ at $z \sim 9$, and attaining $x_{\mathrm{i}, \mathrm{m}} \sim 1$ only by $z \sim 6.5$.

The evolution of the neutral fraction in our model is consistent with, although not required by the measurements of e.g. Fan et al. (2005), which demand only that the IGM reionize sometime before $z \gtrsim 6$. Our model produces an electron scattering optical depth of $\tau_{e}=0.06$, on the low side of CMB constraints (Page et al. 2006), which suggest $\tau_{e}=0.09 \pm 0.03$. We emphasize that our choice of $c(\$ 2.2$ was calibrated so that reionization ends slightly above $z \gtrsim 6$, so this should be viewed as a consequence of our assumptions, rather than a theoretical prediction. Although our model is tuned to give late reionization, analytic models find that the size distribution of HII regions depends primarily on the bias of the ionizing sources, with only an implicit dependence on redshift (Furlanetto et al.|2006a). The size distribution of HII regions at a given ionization fraction is therefore expected to be a robust result, independent of our detailed assumptions about the efficiency of the ionizing sources, (although see Furlanetto et al. 2006a, $\$ 5$ for caveats).

Note that our simulation terminates slightly before reionization completes $\left(x_{i}(z) \sim 1\right)$. We stop our calculation early because we do not include a 'diffuse background' in our simulation (Sokasian et al. 2002), and so our calculation becomes very expensive at the end of reionization when rays wrap around the simulation box several times. In any event, the tail end of reionization is likely poorly modeled in our simulation, since this stage may be regulated primarily by Lyman limit systems (Miralda-Escudé et al. 2000; Furlanetto \& Oh 2005), which are missing in our analysis.

In our simulation, the mass-weighted ionization fraction closely tracks the cumulative number of ionizing photons per Hydrogen atom emitted by our ionizing sources (see the solid red line in Figure 2), but this is partly an artifact of the poor resolution of our radiative transfer calculation. Our low grid resolution underestimates the amount of small scale structure in the density field, and hence the importance of recombinations. In the future, we intend to model recombinations as 'subgrid physics', accounting for enhancements in the recombination rate owing to unresolved small scale structure (see e.g. Kohler et al. (2005)). Presently, we caution that we are under-estimating the number of ionizing photons per Hydrogen atom required to complete reionization. Furthermore, we expect reionization to be even more extended than in our calculation, since recombinations should slow the growth of HII regions.

\section{NUMERICAL SCHEME BASED ON ANALYTIC CONSIDERATIONS}

As motivated in the introduction, we compare our results with a hybrid scheme inspired by the analytic model of FZH04. In this section, we describe this hybrid model, but also refer the reader to FZH04, Zahn et al. (2005) for more information. The major advantage of our implementation over a purely analytic calculation is that the hybrid scheme, which amounts to a Monte-Carlo realization of the analytic model, can capture the asphericity of HII regions during reionization.

The hybrid scheme starts by considering spheres of varying radius surrounding every point in the IGM. Within each such sphere, we calculate the total ionizing photon yield of the sources, and the total enclosed mass in neutral Hydrogen. In the event that the photon yield in a sphere around a given point exceeds the number of interior Hydrogen atoms, the point is considered ionized. FZH04 show that this amounts to a barriercrossing problem, solvable with tools from the excursion set formalism (Bond et al. 1991). Specifically, we assume that the mass contained in halos in a region of total mass $\mathrm{m}$, and over-density $\delta_{m}$, follows the extended PressSchechter formula for the collapse fraction (e.g. Lacey \& Cole 1993):

$$
f_{\text {coll. }}\left(m \geq m_{\min } \mid \delta_{m}, z\right)=\operatorname{erfc}\left[\frac{\delta_{c}(z)-\delta_{m}}{\sqrt{2}\left[\sigma_{\min }^{2}-\sigma^{2}(m)\right]}\right] .
$$

Here $\sigma^{2}(m)$ is the (present day) linear variance of density fluctuations on the scale $m, \delta_{c}(z)=1.686 / D(z)$ is the critical density for collapse scaled to today, and $D(z)$ is the linear growth factor. The quantity $\sigma_{\min }^{2}$ is the linear variance smoothed on a mass scale corresponding to that of the minimum mass halo that can host ionizing sources, presently $m_{\min }=2 \times 10^{9} M_{\odot}(\$ 2.1)$.

For constant mass to light sources, the criterion for a region to self-ionize is then:

$$
\alpha \int_{0}^{t} d t^{\prime} f_{\text {coll. }}\left(m \geq m_{\min } \mid \delta_{m}, t^{\prime}\right) \geq 1,
$$

where $\alpha$ is an efficiency factor linking halo mass and ionizing photon yield. A region can self-ionize if it is sufficiently overdense to satisfy the inequality in Equation (2). Note that this is a slight modification from FZH04 to the case of ionizing sources with a constant mass to light ratio, as assumed in our simulation. In practice, however, we find that the threshold criterion of Equation (2) gives quantitatively similar results to that of FZH04, although it produces slightly larger HII regions.

Our hybrid scheme then amounts to smoothing the linear density field generated from the initial conditions of our N-body simulation, and checking whether cells satisfy the condition of Equation (2). Algorithmically, we start by considering large spheres (comparable to the size of our simulation box), and gradually stepping down in radius, eventually reaching smoothing scales comparable to that of our simulation pixels. At each radius we keep track of which cells satisfy the condition of Equation (2). In the event that a cell does not cross the barrier, 1.e. satisfy the condition of Equation (2), at any smoothing scale, the cell is considered neutral. Proceeding from large smoothing scales and progressing downward to smaller smoothing scales ensures that we account correctly for cells that are ionized by neighboring sources (FZH04). At this stage, we have a map of the ionization field consisting of ' 1 's (completely ionized pixels), and '0's 
(completely neutral pixels). This is a good approximation to the true equilibrium ionization fractions, which are expected to be very close to unity.

This scheme is quite fast: for our present $256^{3}$ grid calculation, with 50 logarithmic smoothing steps, the computation (at a given redshift) takes only $\sim 12$ minutes on a desktop computer with a $3 \mathrm{GHz}$ processor. This is vastly more efficient than our full radiative transfer calculation: our N-body simulation takes 38 hours to run down to $z \sim 6$ using $1342 \mathrm{GHz}$ processors, and our postprocessing calculation requires a few additional days of running time on a large memory computer. With our rapid numerical scheme, we can produce an ionization map based on the analytic model and compare with our radiative transfer simulations. Using precisely the initial conditions from our N-body simulation in our hybrid calculation allows us to compare radiative transfer and analytic ionization fields on a cell-by-cell basis.

Before presenting this comparison, there are a few more pertinent technical details. Ideally, we would compare the analytic and radiative transfer calculations with identical assumptions regarding the ionizing efficiency of our sources, i.e. we should calibrate $\alpha$ in Equation (2) based on the source prescription of $\$ 2.2$. In practice there are several difficulties with matching precisely the simulated source prescription. Most important, Equation (11) is derived assuming sharp $k$-space filtering, while our smoothing procedure adopts a spherical top-hat in real space. This slight inconsistency in our modeling means that our model does not conserve photons precisely, affecting the ionization fraction for a given source efficiency, $\alpha$ (see the Appendix). Further, our simulated mass function is closer to the Sheth-Tormen fitting formula (Sheth \& Tormen 1999) than the Press-Schechter (Press \& Schechter 1974) mass function, and we require an analogue of Equation (1) for the Sheth-Tormen mass function (Barkana \& Loeb 2004b; Furlanetto et al. 2006a). We improve on some of these shortcomings in 75. The upshot of this is that, in order to compare with our radiative transfer simulations, we adjust $\alpha$ in Equation (2) at each redshift to match the (volume-weighted) ionization fraction. This readjustment is usually of order $20 \%$.

We show examples of the resulting ionization maps in Figure 3. The left column shows thin slices through the radiative transfer simulation at three different stages in the reionization process: $z=8.16,7.26$ and 6.89 when the (volume-weighted) ionization fraction is $x_{\mathrm{i}, \mathrm{v}}=$ $0.11,0.33$, and 0.52 respectively. The right column shows corresponding slices from the hybrid simulation scheme. Several conclusions are immediately apparent.

First, the ionized regions are quite large at the intermediate and late stages of reionization. The ionizing sources are highly clustered, and HII regions quickly start growing collectively around the sources, rapidly reaching much larger sizes than can be achieved by individual sources (FZH04) 8 . Second, the hybrid simulation is in good general agreement with the radiative transfer simulation. The hybrid scheme seems to 'locate' the

8 The size of an HII region belonging to an individual average source would be too small to display in this Figure, e.g. for a $10^{10}$ $M_{\odot}$ source shining for one simulation time step of $5 \cdot 10^{7}$ years this size would be only $0.3 \mathrm{Mpc} / \mathrm{h}$. radiative transfer

analytic constant $M / L$

$\mathrm{z}=\mathbf{8 . 1 6}$
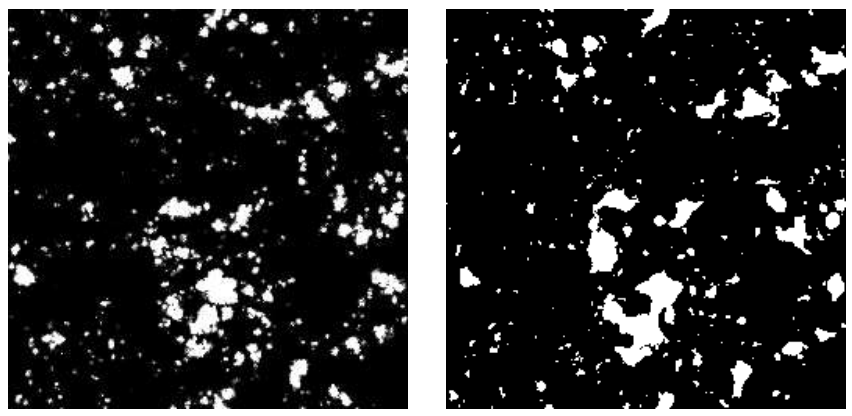

$\mathrm{Z}=7.26$
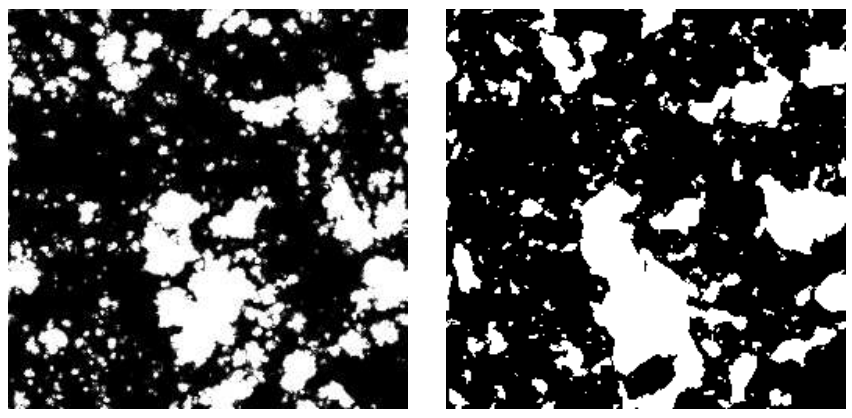

$\mathrm{Z}=6.89$
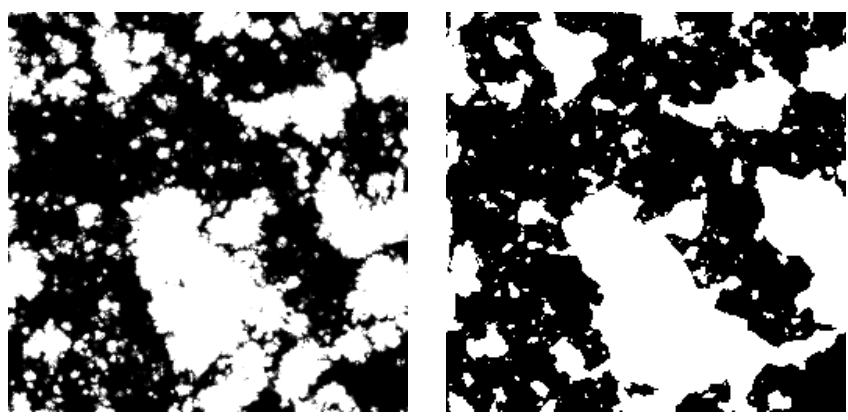

FIG. 3.- Maps of the ionization field. The left column shows HII regions for a thin slice through our radiative transfer simulations at redshifts $\mathrm{z}=8.16, \mathrm{z}=7.26$, and $\mathrm{z}=6.89$ (top to bottom). The volume-weighted ionization fraction at these redshifts is $x_{\mathrm{i}, \mathrm{v}}=$ $0.11,0.33$ and 0.52 , respectively. The slices are $0.25 \mathrm{Mpc} / h$ deep, and $65.6 \mathrm{Mpc} / h$ on a side. The right panel shows the same using our hybrid simulation scheme, as applied to the initial conditions used in our radiative transfer simulation. The analytic modeling agrees well with the more detailed simulation, although there is more small scale structure in the map from the radiative transfer simulation (see text).

HII regions found in the radiative transfer calculation, and additionally reproduces their general morphology. Third, the HII regions in the analytic calculation are a bit more 'connected' than those in the radiative transfer simulation. Equivalently, the ionization field in the radiative transfer simulation appears to have more small scale structure than the ionization field from the hybrid scheme. In the following sections, we will quantify the visual comparison of Figure 3, diagnose differences found, and refine our numerical scheme. We contrast the morphology seen here with that from Iliev et al. (2005) in $\$ 5$.

\section{STATISTICAL DESCRIPTION}


In this section we present a detailed statistical description of our results. Throughout we will compare with our hybrid scheme rather than the purely analytic calculations for two reasons. First, there are technical difficulties in the analytic calculations at intermediate ionization fractions (McQuinn et al. 2005), and second, we would like to be able to model non-spherical bubble shapes.

\subsection{The Bubble PDF}

The first statistic we consider is the probability distribution of bubble sizes. That is, we calculate how large the HII regions are at different stages of reionization. This depends somewhat on how one chooses to define contiguous ionized volumes - Figure 3 clearly illustrates that the ionized regions are not spherical, particularly at the end of reionization. The ionized regions do, however, obtain a reasonably well-defined characteristic size at each redshift. In order to quantify this, we require a convenient and well-motivated definition of 'bubble' that we can apply consistently to the radiative transfer simulation and the hybrid scheme.

Here we adopt a definition of bubble size inspired by the excursion set formalism, upon which our analytic calculation is based (see Iliev et al. 2005 for an alternate approach). Specifically, we 'draw' spheres around each point in our simulation box of varying radius, $R$, and average (smooth) the ionization field within each such sphere. We start by considering large spheres, of volume comparable to that of our simulation box, and step downward in size until we eventually get to the size of our simulation pixels. At each smoothing radius, $R$, we compare the average ionization in each sphere to a threshold ionization, $x_{\mathrm{th}}$. A pixel is marked as 'ionized' and belonging to a bubble of radius $R$, when $R$ is the largest smoothing radius at which the pixel's smoothed ionization exceeds the threshold ionization, $x_{\mathrm{th}}$. If a given pixel fails to exceed the threshold ionization at all smoothing scales, it is considered neutral (not ionized).

The bubble pdf is then derived by tabulating the fraction of ionized pixels that lie within bubbles with radius between $R$ and $R+d R$. With this convention, the bubble pdf is normalized to unity rather than to the mean ionization fraction. The results of this calculation are shown in Figure 4, for an ionization threshold of $x_{\mathrm{th}}=0.9$. The figure illustrates quantitatively the visual impression of Figure 3: the HII regions have a well-defined characteristic size at each stage of reionization, and this characteristic scale evolves as bubbles around neighboring sources overlap and grow collectively (FZH04, Furlanetto et al. 2006a). The characteristic scale evolves from sub-Mpc scales at $z=8.16$, when the volume-weighted ionization fraction is $x_{\mathrm{i}, \mathrm{v}}=0.11$ to $R \gtrsim 10 \mathrm{Mpc}$ co-moving at $z=6.56$ when the volume-weighted ionization fraction is $x_{\mathrm{i}, \mathrm{v}}=0.77$. The large size of HII regions at high ionization fraction implies that large volume simulations are required to adequately sample this stage of reionization (Barkana \& Loeb 2004b, FZH04, Iliev et al. 2005). The precise value of the characteristic bubble size depends somewhat on the number we adopt for the threshold ionization. For instance, if we instead adopt the less stringent threshold of $x_{\mathrm{th}}=0.7$, the characteristic size increases by a factor of $\sim 2$ near $z=8.16$. Again, while our definition of bubble-size is somewhat arbitrary, the bubbles nevertheless have a well-defined characteris-

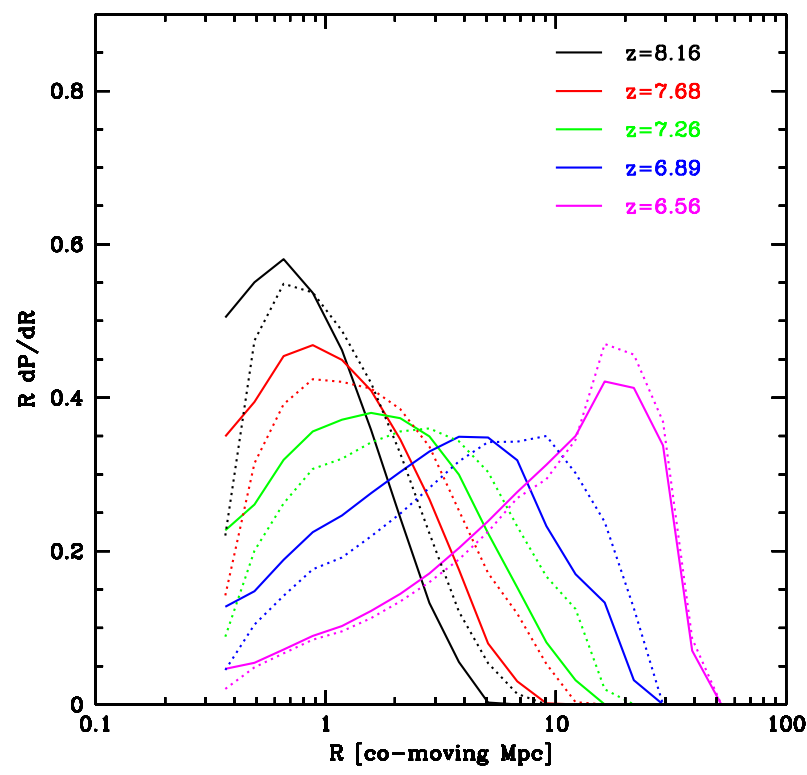

FIG. 4.- Size distribution of HII regions as a function of redshift. The solid curves show results from the radiative transfer simulation, while the dotted curves are from the analytic calculation. We adopt a threshold ionization of $x_{\mathrm{th}}=0.9$ (see text). The volume-weighted ionization fractions at the redshifts shown are $x_{\mathrm{i}, \mathrm{v}}=0.11,0.20,0.33,0.52,0.77$ at $z=8.16,7.68,7.26,6.89$ and $z=6.56$ respectively.

tic scale (Furlanetto et al. 2006a), and our algorithm can be applied consistently to each of the analytic model and radiative transfer ionization maps.

The dotted lines indicate that our hybrid scheme reproduces the bubble pdf simulated through radiative transfer quite accurately, roughly matching the characteristic bubble size and its trend with redshift. The hybrid scheme however leads to slightly larger HII regions at all but the final redshift. We will discuss this difference in future sections. At the final redshift, the agreement is almost exact, however here our simulated volume is too small to provide a representative sample.

\subsection{Power Spectra of the ionized fraction}

For further comparison, we measure the (spherically averaged) $3 \mathrm{~d}$ ionization power spectrum as a function of redshift. We consider the ionization field $\delta_{x}=x(\vec{r})-\langle x\rangle$, where $x(\vec{r})$ denotes the ionization at spatial position $\vec{r}$, and $\langle x\rangle$ denotes the volume-averaged ionization. Note that we do not normalize by the mean ionization here, i.e. we consider the absolute ionization fluctuation, rather than the fractional fluctuation. The result of the power spectrum calculation is shown in Figure 5, with power spectra calculated from the radiative transfer simulation plotted in red. Throughout this paper we plot the dimensionless power spectrum, $\Delta^{2}(k)=k^{3} P(k) /\left(2 \pi^{2}\right)$, which yields the contribution to the variance per logarithmic interval in $k$. On large scales at high redshift the ionization power spectrum is proportional to the density power spectrum, while it turns over or flattens on scales in which there are ionized bubbles. On intermediate 


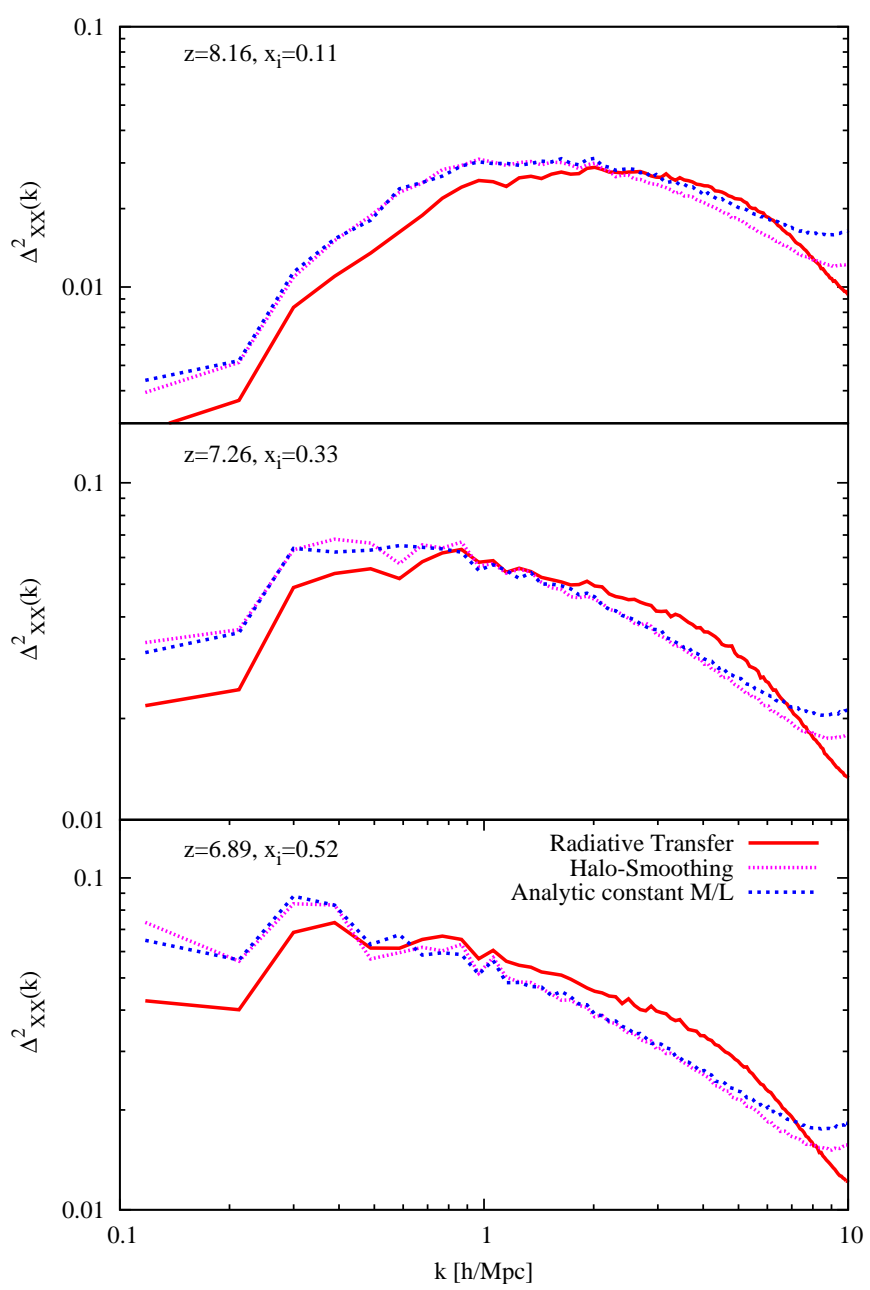

FIG. 5.- Power spectra of the ionized fraction, going from large redshift (small ionization fraction) to small redshift (large ionization fraction). The red lines are from the radiative transfer simulation, the blue dashed lines are from the analytic hybrid calculation, while the purple dotted lines show results from the improved scheme of the next section. The high- $k$ behavior $\left(k \gtrsim 10 h \mathrm{Mpc}^{-1}\right)$ is an artifact from discreteness noise.

scales the power spectrum from the radiative transfer simulation has a somewhat larger amplitude. We attribute this to a superior tracking of the density field around the edges of the bubbles: rays can travel into underdense regions and will be hindered by overdensities. In the numeric schemes the features of the density field are washed out somewhat, resulting into a generally smoother edge structure, see also Figure 3 The bubble 'feature' moves to progressively larger scales (small $k)$ as reionization proceeds, a further illustration of the bubble growth seen in Figure 4 . The blue dashed curves show power spectra from our hybrid simulation, which are similar to the radiative transfer power spectra, except with slightly more large scale power, and slightly less small scale power. One can also infer from the figure that an even larger volume simulation is preferable, in order to better sample the large scale ionization power spectrum. Finally, the purple dotted lines are from an improved numerical scheme which we discuss in the next section.

In order to further quantify the agreement between the radiative transfer simulation and the hybrid scheme, we

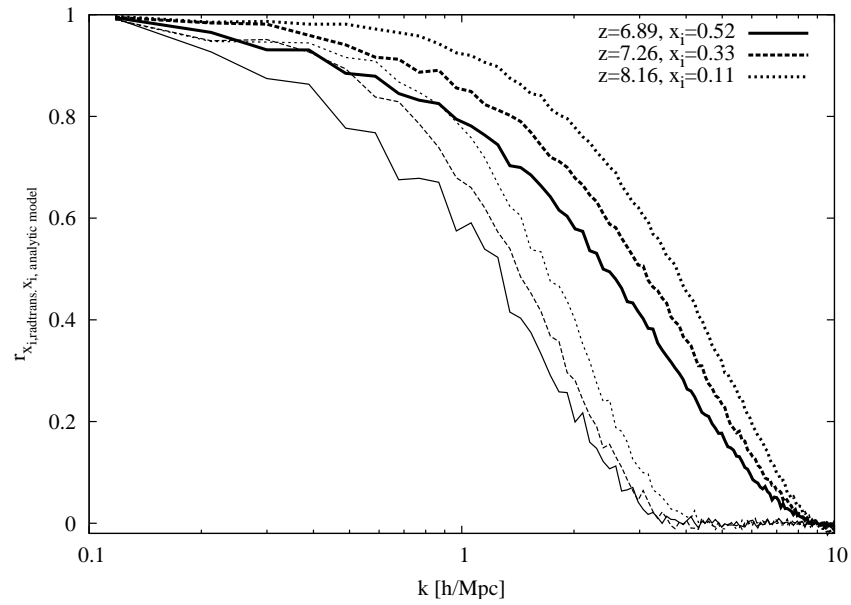

FIG. 6.- Cross correlation coefficient between the ionization fields from the radiative transfer simulation and the analytic model calculations. The thin lines show the cross correlation coefficient between the radiative transfer and hybrid simulations at a few different redshifts. The thick lines show corresponding results from the improved hybrid simulation described in the next section.

calculate the cross correlation coefficient between the two ionization fields. The cross correlation coefficient is defined by $r(k)=\Delta_{\mathrm{x} 1, \mathrm{x} 2}^{2}(k) /\left[\Delta_{\mathrm{x} 1}^{2}(k) \Delta_{\mathrm{x} 2}^{2}(k)\right]^{1 / 2}$. In this equation $\Delta_{\mathrm{x} 1, \mathrm{x} 2}^{2}(k)$ is the cross power spectrum between the radiative transfer simulation and hybrid scheme ionization fields, while $\Delta_{\mathrm{x} 1}^{2}(k)$, and $\Delta_{\mathrm{x} 2}^{2}(k)$ are their respective power spectra. The cross correlation coefficient is bounded between 1 and -1 , with $r(k)=1$ indicating perfectly correlated modes, and $r(k)=-1$ designating perfectly anti-correlated modes. The results of this calculation are shown as thin lines in Figure 6 (ignore, for now, the thick lines which show results from the improved hybrid scheme introduced in the next section). The correlation coefficient is always larger than $r \sim 0.5$ for scales larger than $k \lesssim 1 h \mathrm{Mpc}^{-1}$, while it drops off on smaller scales. This quantifies the qualitative agreement suggested by Figure 3 the radiative transfer and hybrid scheme ionization fields trace each other closely on scales larger than $k \lesssim 1 h \mathrm{Mpc}^{-1}$. The cross correlation between the two fields becomes slightly weaker at low redshift, as the average ionization increase. A plausible explanation for the slightly worse agreement at low redshift is that our hybrid simulation scheme has difficulty with 'bubble mergers' (see the Appendix), which are more frequent at high ionization fraction.

Why does the cross correlation between the two fields drop off around $k \gtrsim 1 h \mathrm{Mpc}^{-1}$ ? The analytic model assumes a one-to-one correspondence between the abundance of halos and the (Lagrangian) matter overdensity on a given smoothing scale. We know this is inexact. For one, the abundance of our minimum mass sources is $M d n / d M \lesssim 1 \mathrm{Mpc}^{-3}$. On $\sim 1 \mathrm{Mpc}$ scales, we therefore expect significant Poisson scatter in the abundance of ionizing sources in our radiative transfer simulation (see also Furlanetto et al. 2006a: Cohn \& Chang 2006). To explore this further, we compute the cross power spectrum between the halo density field and the matter density field. The cross correlation coefficient between the halo and matter density fields qualitatively mirrors the cross 
correlation between the two ionization fields seen in Figure 6, dropping off at $k \gtrsim 1 h \mathrm{Mpc}^{-1}$. In other words, the halo bias is stochastic on scales of $k \gtrsim 1 h \mathrm{Mpc}^{-1}$ for our assumed source population. This stochasticity is not incorporated in our analytic hybrid scheme, and likely leads to the lack of small scale structure compared to the ionization field simulated through radiative transfer. We will return to this issue in \$5. We note here, however, that this Poisson scatter would presumably be less important if our radiative transfer simulation resolved smaller, more abundant galaxies.

The analytic model connects ionized regions with large scale overdensities, which contain more sources and are reionized before underdense regions (FZH04, Barkana \& Loeb 2004b). The model therefore predicts that the ionization field is positively correlated with the matter density (e.g. McQuinn et al. 2005b), before turning over on scales comparable to that of the ionized bubbles (FZH04). Figure 7 shows the cross power spectrum between ionization and density (bottom panel) as well as the cross-correlation coefficient between the two fields. The radiative transfer simulation results (solid lines) nicely mirror the analytic model predictions (dotted lines). Since the analytic model ionization field is based on the initial condition density field, it is slightly less correlated with the evolved density field than the radiative transfer simulation ionization field. In our radiative transfer simulation and hybrid scheme, reionization proceeds inside-out with the overdense regions reionized before underdense regions, as emphasized by FZH04 and Sokasian et al. (2003, 2004). Recombinations, underestimated in our present simulations, could potentially weaken this correlation or, in an extreme case, reverse the correlation with voids ionized first (Miralda-Escudé et al. 2000). We intend to explore this in future work.

\section{IMPROVED NUMERICAL SCHEME}

Although the agreement between our radiative transfer simulation and the hybrid scheme is already quite good, we present here a modified numerical scheme that improves upon the one presented in $\$ 3$ and Zahn et al. (2005). Specifically, we aim to fix two short-comings of the analytic calculation. First, as mentioned previously, the analytic calculation is based on the PressSchechter formula for the collapse fraction. This formula is derived assuming sharp $k$-space filtering, while our scheme filters the initial density field with a top-hat in real space, which is slightly inconsistent (McQuinn et al. 2005, the Appendix). Second, the mass function in our radiative transfer simulation (Figure 1) is closer to the Sheth \& Tormen (1999) mass function than the Press \& Schechter (1974) mass function. Finally, the analytic calculation assumes a one-to-one correspondence between initial over-density and halo abundance. As we discussed in 4.2 , the halo bias in our N-body simulation is stochastic on small scales.

Each of these shortcomings can be remedied by directly using the simulated halos in our numerical scheme, rather than the Press-Schechter formula for the collapse fraction. More specifically, we place the halo distribution from our N-body simulation on a grid and compare, at each grid cell, the halo mass to the total mass enclosed by a spherical top-hat. We then use a condition analogous to Equation (2) to determine whether a region is

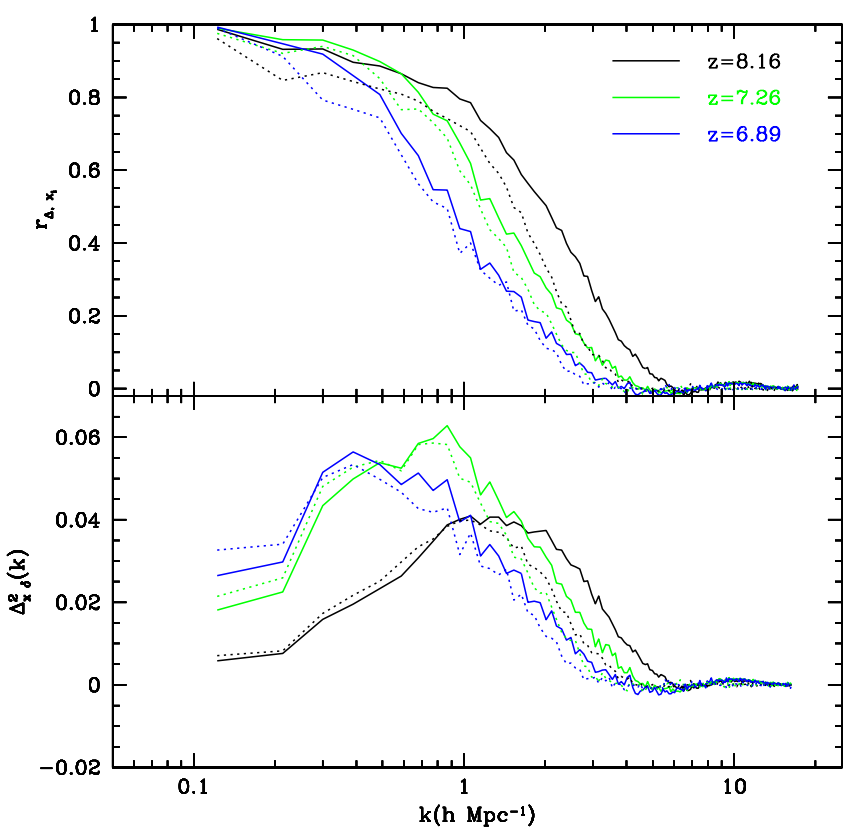

FIG. 7.- Top panel: Cross correlation coefficient between the ionization and density field. The solid (dotted) lines show the cross correlation coefficient between the ionization and density fields in the radiative transfer simulation (hybrid scheme) at several redshifts. Bottom panel: Cross-power spectrum between the ionization and density field. Solid lines are calculations from the radiative transfer simulation, while dotted lines are from the hybrid scheme.

ionized by the sources within it. In other words, the calculation proceeds exactly as in $\$ 3$, except that we use the halo distribution directly from the simulation, rather than Press-Schechter theory. Note further that we now consider the evolved, non-linear density field rather than the initial, linear density field to determine if a region can self-ionize. We will call this improved numerical implementation the 'halo-smoothing' scheme in what follows. The CPU intensity of this scheme is again dominated by the number of FFT's necessary to achieve convergence in the bubble size statistic. As with the analytic scheme, this is roughly 12 minutes on a $3 \mathrm{GHz}$ Intel Xeon desktop computer.

The results of this new scheme are shown in comparison with radiative transfer and analytic calculation in Figure 10, where we show $21 \mathrm{~cm}$ brightness temperature fluctuations (see 86 ) for a thin slice through the simulation volume. This is analogous to Figure 3 except the ionized regions are now dark, the neutral regions now bright, and fluctuations in the gas density are now visible in the neutral regions. The left column shows results from our radiative transfer simulation, the right column shows the standard FZH04-type implementation, while the center column shows our improved halo-smoothing scheme. The blue dots in the left and center column show the ionizing sources contained in the thin simulation slice. The new scheme clearly resembles the full simulation more closely, with more disconnected ionized regions, owing to the presence of Poisson fluctuations in the source distribution.

Figure 5 quantitatively illustrates improved agreement with the radiative transfer calculation, with our im- 
proved scheme showing more small scale power than the hybrid simulation scheme. Figure 6 additionally shows the cross correlation between the radiative transfer ionization field and the ionization field in the improved numerical scheme (thick lines). The halo-smoothing ionization field traces the ionization field simulated through radiative transfer more closely, and down to smaller scales, than in our initial calculation. We attribute the improved agreement largely to our incorporation, in the improved scheme, of Poisson scatter in the halo abundance.

If the ionizing sources are even less abundant than we assume presently, the Poisson scatter naturally becomes more important. Indeed for sufficiently rare sources, Poisson fluctuations dominate over source clustering on the scale of a typical bubble, and bubble growth is less 'collective' than in our fiducial model. In this regime, the morphology of HII regions during reionization may be qualitatively different. To examine this, we repeat our halo-smoothing calculation at $z=7.26$ including only halos with $m \geq 4 \times 10^{10} M_{\odot}$ as sources. We adjust the ionizing efficiency of these rarer sources upward to match our usual ionized fraction at this redshift, $x_{\mathrm{i}, \mathrm{v}}=0.33$, in order to compare maps at fixed ionization fraction. The result of this calculation is shown in the left panel of Figure 8. One can see that the bubbles are considerably more spherical than in our usual source prescription (middle panel), and that the HII regions have a more sharply defined scale. The left panel further illustrates that for this source prescription there are very few sources in each bubble. Note that this is a thin slice, and some sources contributing to bubble growth lie above or below it.

Furthermore, the left panel qualitatively resembles the morphology seen in the reionization simulations of Iliev et al. (2005) (see their Figure $8^{9}$ ). Their simulations are done at higher redshift, but have a similar source number density as our present, extreme choice of $m \geq 4 \times 10^{10} M_{\odot}$ (with this choice our simulation volume contains roughly $5,000$ sources at $\mathrm{z}=7.26)$. We regard the morphology seen in Iliev et al. (2005) as unlikely to represent the true morphology of HII regions during reionization. Their choice of minimum source mass $\left(M_{\min }=2.5 \times 10^{9} M_{\odot}\right)$ is driven by the low mass resolution of their simulations, and the efficiency of their ionizing sources is boosted extremely high in order to match first-year WMAP constraints (Kogut et al. 2003). In other words, their simulation represents a very extreme case of reionization by rare, bright sources. Our simulation is also missing plausible ionizing sources, given our comparable minimum source mass. However, owing to the different assumptions about the ionizing efficiency in our simulation, reionization occurs later and so our sources are much more abundant $(265,000$ sources in the simulation volume at $z=7.26$ ). We are hence still in the regime where HII regions grow collectively, and we expect only small modifications to the morphology and size distribution of HII regions when we include still smaller mass sources. This is illustrated in the right panel of Figure 8 where we show predictions for our original hybrid scheme (\$3), with the minimum source mass extended down to the cooling mass, $M_{\text {min }} \sim 10^{8} M_{\text {sun }}$. While there are some differ-

\footnotetext{
9 Note for comparison with these authors' figure: their simulation box has a side length of $L=100 \mathrm{Mpc} / h$, while ours has $L=65.6 \mathrm{Mpc} / h$.
}

ences with the results from our usual source prescription (center panel), the differences are clearly smaller than in comparison to the Poisson-dominated case (left panel). 10 The differences with Iliev et al. (2005) highlight the utility of our fast numerical schemes for quickly examining many different prescriptions for the ionizing sources and for understanding the robustness of the results.

\section{21 CM SIGNAL AND POWER SPECTRA}

The statistics discussed in $\$ 4$ are largely diagnostic, aimed at describing the size distribution of HII regions in the simulation, and characterizing the agreement between the radiative transfer simulation and analytic calculations. In this section we make a more observationally relevant comparison, contrasting radiative transfer and analytic $21 \mathrm{~cm}$ power spectra.

The $21 \mathrm{~cm}$ brightness temperature, relative to the CMB, at observed frequency, $\nu$, and redshift, $z$, is (e.g. Zaldarriaga et al. 2004):

$$
\begin{aligned}
\delta T(\nu) \approx & 26\left(1+\delta_{s}\right) x_{H}\left(\frac{T_{S}-T_{\mathrm{CMB}}}{T_{S}}\right)\left(\frac{\Omega_{b} h^{2}}{0.022}\right) \\
& \times\left[\left(\frac{0.15}{\Omega_{m} h^{2}}\right)\left(\frac{1+z}{10}\right)\right]^{1 / 2} \mathrm{mK} .
\end{aligned}
$$

where $\delta_{s}$ is the density contrast of gas in redshift space, and $T_{S}$ is the spin temperature of neutral hydrogen. At the redshifts we consider presently, the $21 \mathrm{~cm}$ excitation temperature is likely coupled to the gas temperature, and much larger than the temperature of the CMB (e.g. Furlanetto 2006, Chen \& Miralda-Escude 2004; Ciardi \& Madau 2003), $T_{S}>>T_{\mathrm{CMB}}$, implying $\delta T \propto\left(1+\delta_{s}\right) x_{H}$. We then model the $21 \mathrm{~cm}$ brightness temperature using the simulated density and peculiar velocity fields, in conjunction with radiative transfer/analytic calculation simulated ionization fields. We incorporate here the effect of redshift space distortions, taking into account the simulated peculiar velocity field. On large scales, linear infall boosts the spherically averaged $21 \mathrm{~cm}$ redshift power spectrum relative to its real space analogue, analogous to the 'Kaiser effect' in galaxy surveys (Kaiser 1987; Bharadwaj \& Ali 2004, McQuinn et al. 2005; Barkana \& Loeb 2005). By spherically averaging the signal we lose information about the ionizing sources as well as cosmological parameters, as was discussed e.g. in Barkana \& Loeb (2005). However, the first generation $21 \mathrm{~cm}$ experiments will be sensitive mainly in the frequency direction and have difficulty measuring the full angular dependence of the signal (McQuinn et al. 2005).

The result of our power spectrum calculation is shown in Figure 9 for three different redshifts during reionization. The results are qualitatively similar to those of Figure 5 , and can be roughly understood by decomposing the $21 \mathrm{~cm}$ power spectrum into three constituent pieces (FZH04):

$$
\Delta_{21}^{2}(k)=\bar{T}_{b}^{2}\left[\Delta_{\mathrm{xx}}^{2}(k)-\frac{8}{3} \bar{x}_{H} \Delta_{\mathrm{x} \delta}^{2}(k)+\frac{28}{15} \bar{x}_{H}^{2} \Delta_{\delta \delta}^{2}(k)\right] .
$$

10 We note the possibility that feedback effects, which have note been included in our simulations, might suppress the formation of the lowest mass sources and lead in extreme cases to a morphology that resembles that seen in Iliev et al. (2005). 

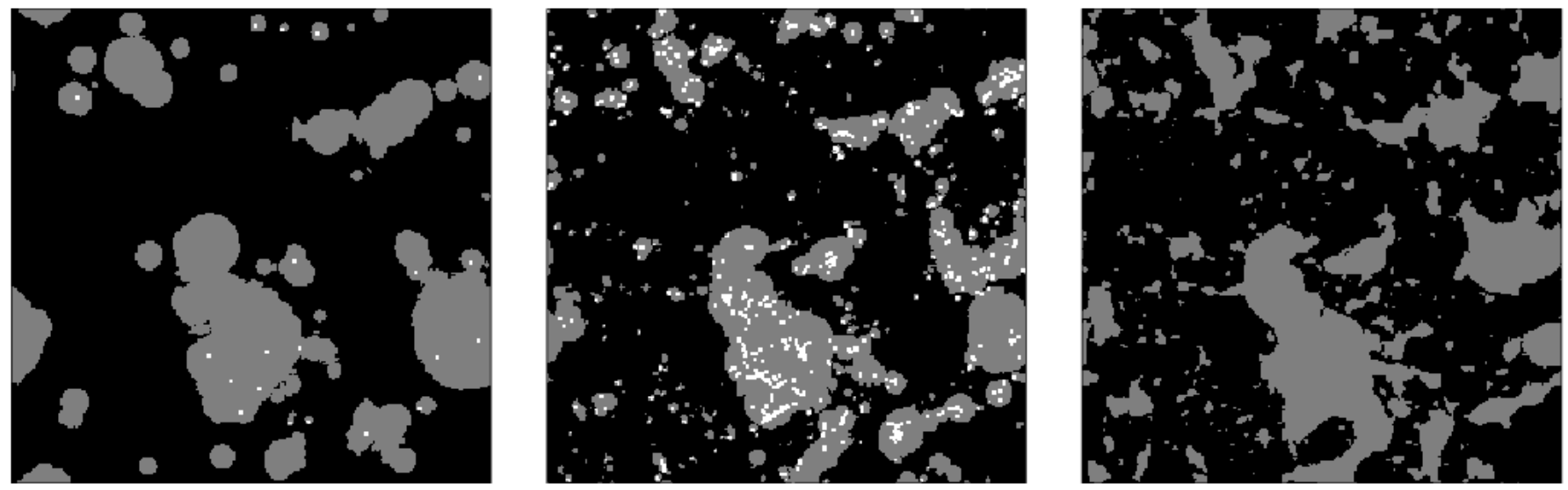

FIG. 8.- Dependence of reionization morphology on source density. In the left panel we show the ionization field from our halo-smoothing procedure using only sources (white points) with mass larger than $M \geq 4 \times 10^{10} M_{\odot}$ (note that some sources contributing to the ionized regions lie in front or behind the thin slice shown). With this choice, the number density of sources roughly matches that of $M \geq 2 \times 10^{9} M \odot$ sources at $z \sim 14$ (as in Iliev et al. 2005). The center panel shows the result with our usual source prescription, indicating a significantly more complex morphology. Finally the right panel shows, for comparison, the analytic model with $M_{\text {min }}=10^{8} M_{\odot}$. Each panel is at $z=7.26$, and in each case the source efficiencies are adjusted to match $x_{\mathrm{i}, \mathrm{v}}=0.33$.

Here $\Delta_{\mathrm{xx}}^{2}$ refers to the ionization power spectrum, $\Delta_{\mathrm{x} \delta}^{2}$ refers to the ionization-density cross power spectrum, and $\Delta_{\delta \delta}^{2}$ refers to the density power spectrum. Note that, for illustrative purposes we ignore higher order terms (McQuinn et al. 2005, Furlanetto et al. 2006a), although their effects are included in our calculations. The numerical coefficients in this decomposition come from angleaveraging the redshift space power spectrum. On scales much larger than the size of the ionized bubbles, each term in this decomposition is directly proportional to the density power spectrum, and so the $21 \mathrm{~cm}$ power spectrum is directly proportional to the density power spectrum. On the other hand, on very small scales one would expect that the $21 \mathrm{~cm}$ power spectrum approaches the density power spectrum multiplied by the neutral fraction squared (and a constant factor $\simeq 1.87$ for the spherically averaged redshift space case). The latter is shown in the thin dashed curves in the Figure. The discrepancy seen is due to the significance of higher order terms that were neglected in Equation 3, that in reality amount to corrections of order one (Lidz et. al 2006).

These qualitative trends can be seen in Figure 9. For further illustration, we extrapolate our predictions to large scales using an analytic model hybrid simulation (green long-dashed lines) which we based on a Gaussian random field with sidelength $300 \mathrm{Mpc} / \mathrm{h}$.

At high redshift, where the ionized regions are small, the $21 \mathrm{~cm}$ power spectrum has the shape of the density power spectrum. At lower redshifts, it begins to flatten on large scales owing to the presence of ionized regions, before following the shape of the density power spectrum again on small scales. This flattening moves to progressively larger scales as reionization proceeds, and the bubbles grow larger. Our first observational handle on the characteristic sizes of HII regions at different stages of reionization will likely come from measuring the $21 \mathrm{~cm}$ power spectrum, and observing this flattening. In other work, we will explore the extent to which the size distribution of HII regions can be extracted from future measurements of the $21 \mathrm{~cm}$ power spectrum (Zahn et al. 2006).

Notice that the agreement between the analytic and radiative transfer $21 \mathrm{~cm}$ power spectra is even better than the agreement between the ionization power spectra. While the ionization field in the radiative transfer simulation has more small scale power than the analytic model ionization field, the different approaches show similar amounts of small scale $21 \mathrm{~cm}$ power. This owes to the small-scale dominance of the $\Delta_{\delta \delta}^{2}(k)$ term in the 21 $\mathrm{cm}$ power spectrum, which overwhelms the difference in small scale ionization power (see Figure 5). The $21 \mathrm{~cm}$ power spectrum in each analytic scheme seems to provide a very good approximation to the results of our full radiative transfer simulations. Some of the difference on large scales may be attributable to our limited simulation volume, and a convergence test with increasing boxsize would be informative, but we leave this to future work.

\section{CONCLUSIONS}

We have presented results from a large volume radiative transfer simulation and fast numerical schemes based on analytic considerations, and given a detailed comparison. Our basic conclusion is that the approximate schemes agree remarkably well with the radiative transfer simulation.

Future work should investigate the effect of recombinations which, we anticipate, will lead to two primary modifications (Furlanetto \& Oh 2005). First, recombinations will slow down reionization by requiring more ionizing photons to achieve a given ionization fraction. This should mainly act to modify the redshift evolution of the ionization fraction, and not the size distribution of HII regions at a given ionization fraction, our main focus in the present work. Second, ionization fronts may be halted upon impacting dense clumps, where the recombination rate is very high (e.g. Miralda-Escudé et al. 2000; Shapiro et al. 2004; Furlanetto \& Oh 2005). 'This latter effect might, indeed, modify the size distribution of HII regions at a given ionization fraction. However, as long as mini-halos are destroyed by pre-heating prior to reionization (e.g. Oh \& Haiman 2003), estimates show this effect is important only at the tail end of reionization, when $x_{\mathrm{i}, \mathrm{v}} \gtrsim 0.77$ (Furlanetto \& Oh 2005), which we do not presently simulate. 


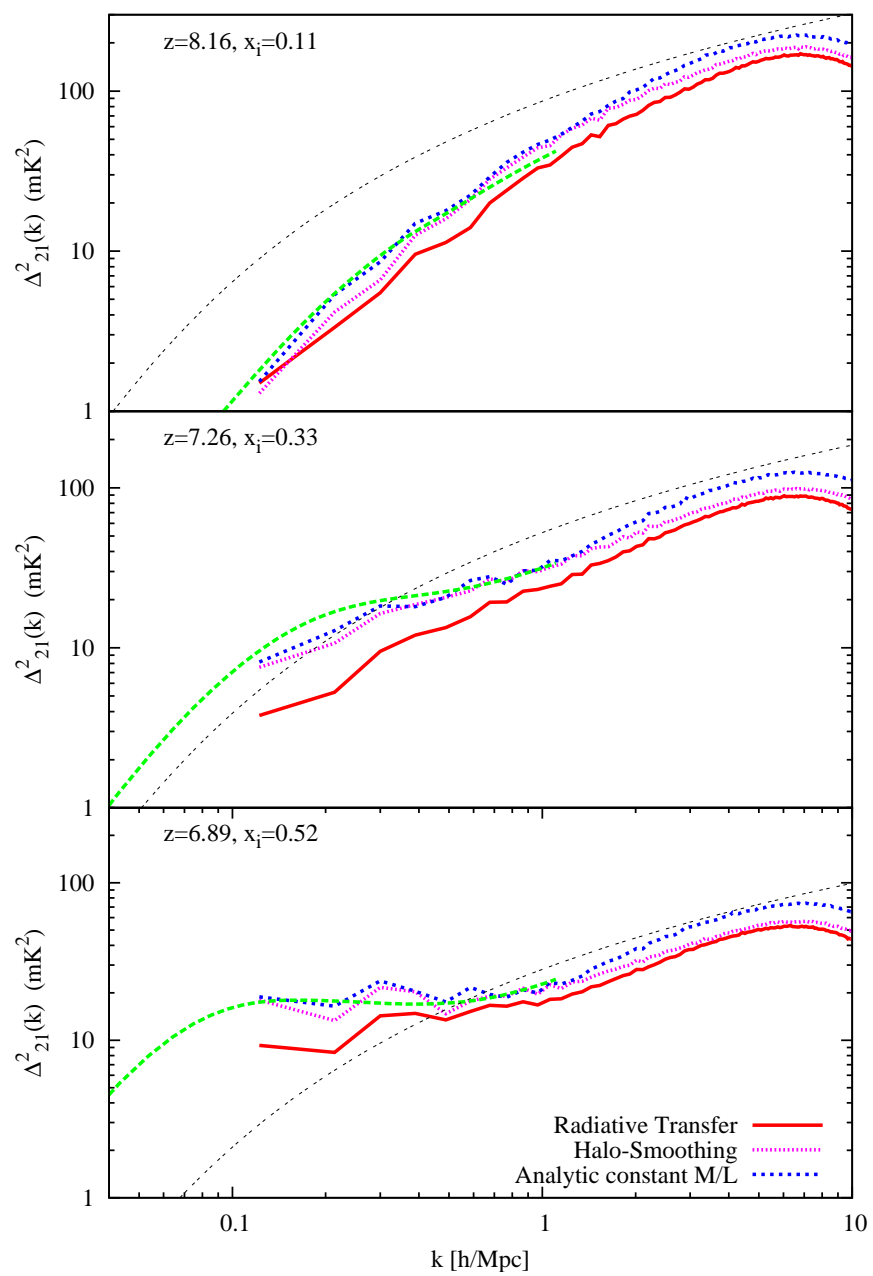

FIG. 9.- The $21 \mathrm{~cm}$ brightness temperature power spectra in redshift space. The solid red, short-dashed blue and dotted purple lines show the radiative transfer, analytic, and halo-smoothing power spectra, respectively. The green long-dashed lines show extrapolations of the analytic predictions to large scales. Some of the differences in the predictions on large scales may be attributable to our limited simulation volume. The redshift space $21 \mathrm{~cm}$ power spectrum approaches $P_{\delta} x_{H}^{2} 1.87$ (shown in the thin dashed curve) on small scales. The differences seen are due to the relevance of higher order contributions to the $21 \mathrm{~cm}$ power spectrum (see upcoming work).

In the future we will address these issues explicitly, along with other refinements to our radiative transfer simulations. We intend to consider a more sophisticated prescription for the ionizing sources (Springel \& Hernquist 2002: Sokasian et al. 2003), and extend the mass range of our sources down to the cooling mass. It will be interesting to examine how sensitive the $21 \mathrm{~cm}$ predictions are to the assumed properties of the ionizing sources (Furlanetto et al. 2006a). In particular, in \$5 we found that the morphology and size distribution of HII regions differs dramatically from our fiducial model when extremely rare, bright sources dominate. This warrants further quantitative investigation. Finally, we intend to examine the effect of feedback on reionization, incorporating Jeans mass suppression (e.g. Barkana \& Loeb 2000; Babich \& Loeb 2005; Kramer et al. 2006) in reionized regions of the IGM (McQuinn et al. 2006).

In spite of these refinements, we contend that the agreement demonstrated in this paper illustrates that the analytic models are on the right track, and provide a useful complementary tool to radiative transfer simulations. The approximate schemes described here are very fast, allowing quick coverage of a large parameter space, convenient for forecasting constraints from upcoming $21 \mathrm{~cm}$ surveys (Zahn et al. 2006). Even full radiative transfer simulations currently have a large number of free parameters related to the efficiency of the ionizing sources, the escape fraction of ionizing photons, and sub-grid clumping. Our numerical schemes allow one to gauge how the expected signal depends on these numerous, unconstrained parameters. It can also be used to investigate non-Gaussianities in the $21 \mathrm{~cm}$ signal, as advocated by Furlanetto et al. (2004b), and to construct mock $21 \mathrm{~cm}$ survey volumes, providing a useful test of data analysis procedures, which are presently still under development. This is particularly relevant given that surveys like the MWA will be done in large volumes of several co-moving cubic Gigaparsecs, prohibitive for current radiative transfer simulations, but manageable with analytic calculations. Finally, it might be interesting to couple the fast analytic model schemes with a gas-dynamical calculation to investigate the impact of reionization on galaxy formation.

\section{ACKNOWLEDGMENTS}

We thank Aaron Sokasian for providing his radiative transfer code, and Volker Springel for providing an enhanced version of Gadget. We also thank Tom Abel, Marcelo Alvarez, Katrin Heitmann, Salman Habib, and Miguel Morales for helpful conversations. The authors are supported by the David and Lucile Packard Foundation, the Alfred P. Sloan Foundation, and NASA grants AST-0506556 and NNG05GJ40G.

\section{APPENDIX}

\section{PHOTON CONSERVATION IN OUR APPROXIMATE SIMULATION SCHEMES}

The objective of this Appendix is to show that the pure FZH04 model conserves photons, but that our numerical schemes do not precisely conserve photons. We then discuss the implications of this finding. In the pure FZH04 model, we can prove that the global ionization fraction is given by $\bar{x}=\zeta \times f_{\text {coll. }}$. This is just a reflection of photon conservation: as we sum up the total ionized mass from individual HII regions, no photons are lost or gained in our accounting of the net ionized mass.

A rigorous proof proceeds as follows. For simplicity, we outline this proof using the pure FZH04 barrier, but the proof can be easily generalized to the barrier of Equation (2). Let us consider random walks in the $\left(\delta, \sigma^{2}\right)$ plane (e.g. Bond et al. 1991), generated using top-hat smoothing in $k$-space. We consider the first up-crossing distributions for two types of barriers. First, we examine the probability that a random walk crosses the 'bubble barrier', representing the critical density threshold for a region to self-ionize (see Figure 1 of FZH04). We denote the differential probability 
radiative transfer

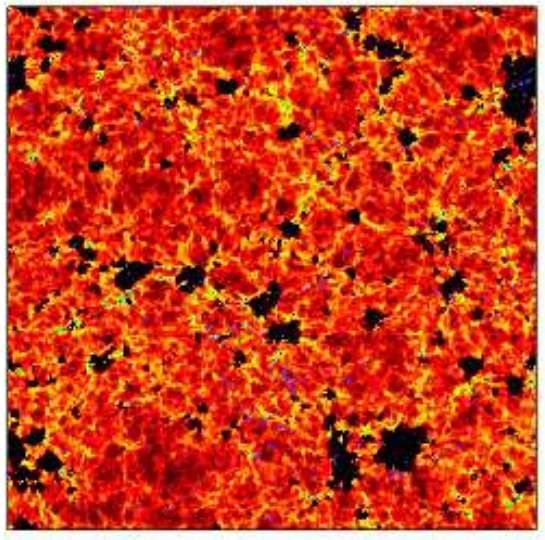

halo-smoothing

$z=8.16$

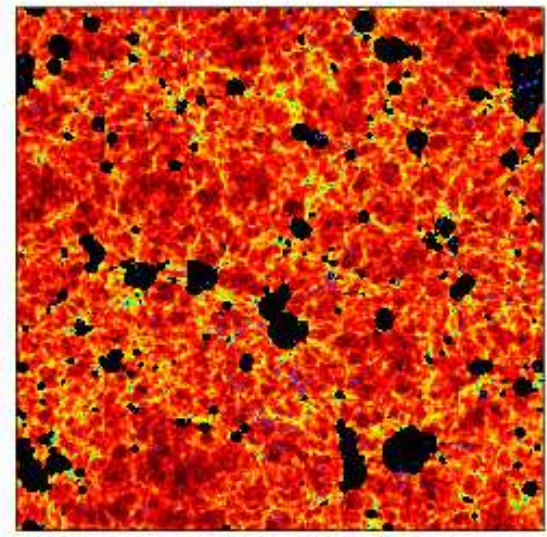

$\mathrm{z}=\mathbf{7 . 6 8}$
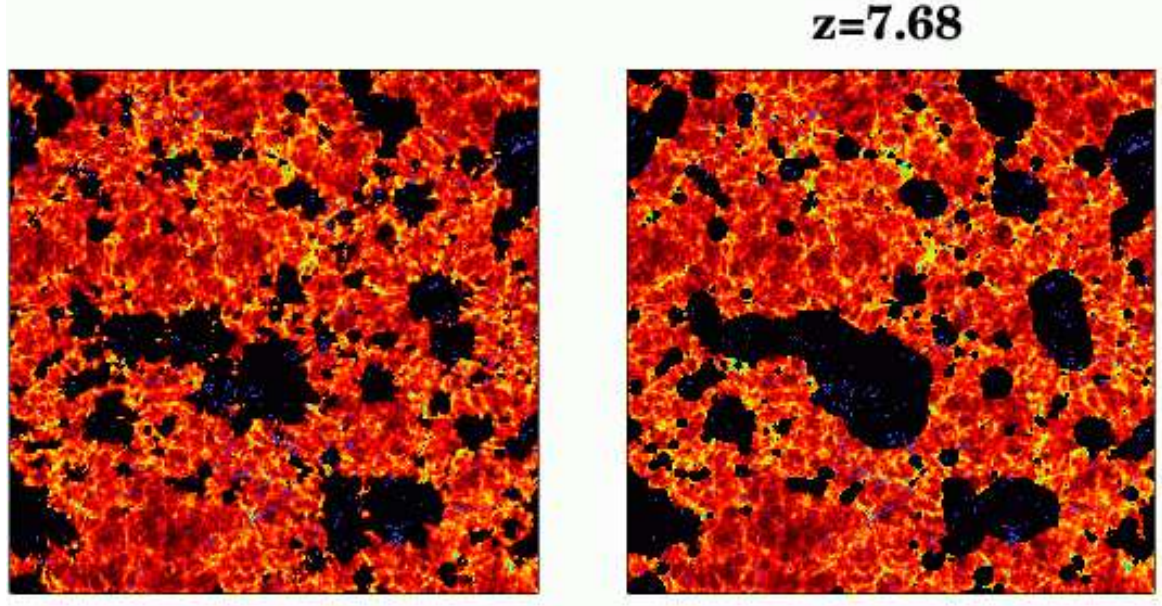

analytic constant $M / L$

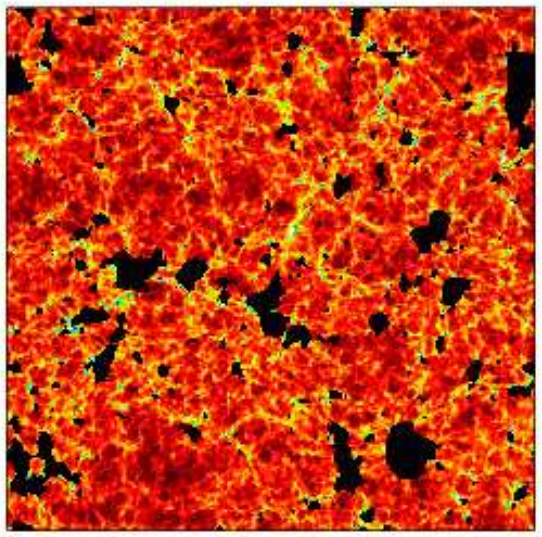

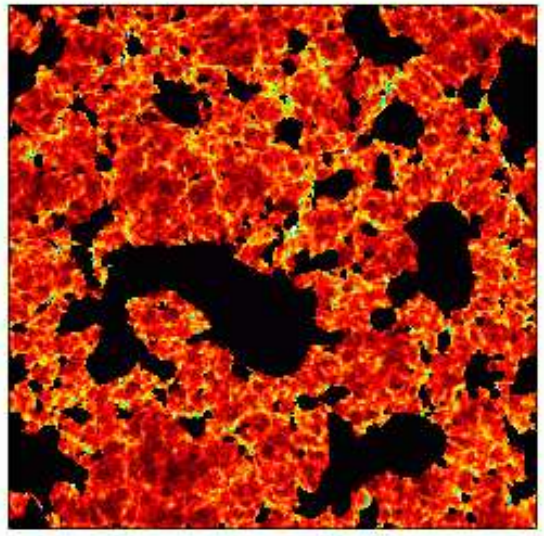

\section{$\mathrm{Z}=6.89$}
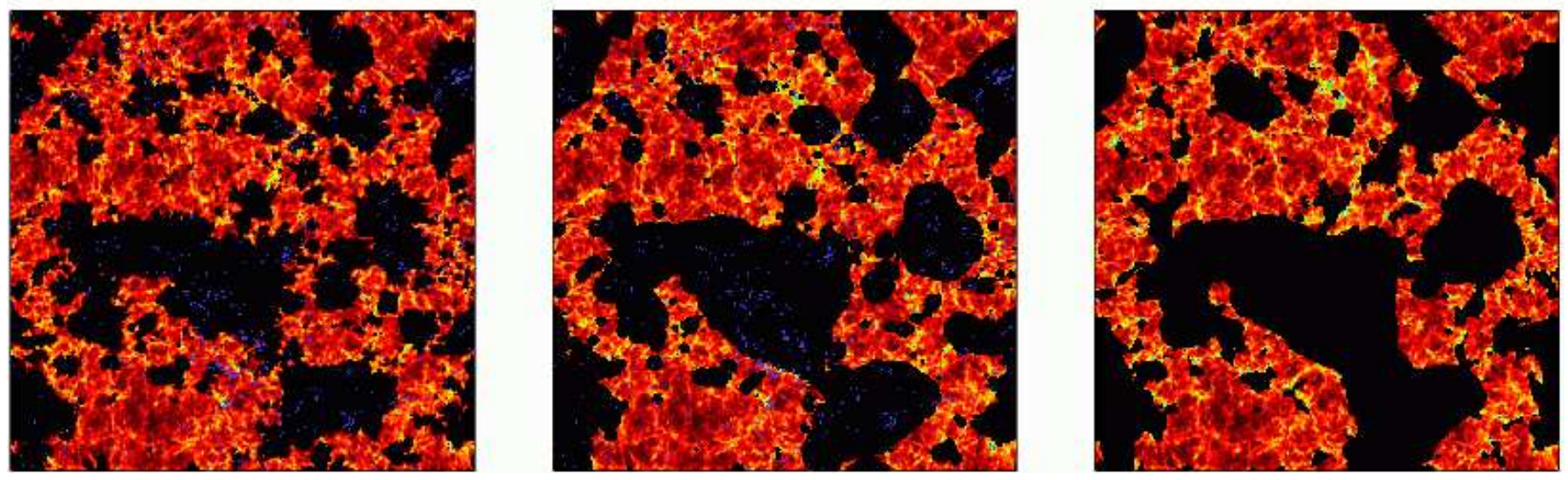

FIG. 10. - $21 \mathrm{~cm}$ brightness temperature fluctuations. We compare $21 \mathrm{~cm}$ maps from the radiative transfer simulation and numerical scheme at three different redshifts. Each map is $65.6 \mathrm{Mpc} / h$ on a side, and $0.25 \mathrm{Mpc} / h$ deep, comparable to the frequency resolution of planned experiments, and shows a different cut then Figure 3 The ionized fractions are $x_{\mathrm{i}, \mathrm{V}}=0.11,0.33$ and 0.52 for $z=8.16,7.26$ and 6.89 respectively. Left column: Radiative transfer calculation with ionizing sources (blue dots). Middle column: Halo-smoothing procedure (see text) with sources/halos from the N-body simulation. Right column: Constant mass-to-light ratio version of FZH04, based purely on the initial, linear dark matter overdensity. 
that a random walk crosses this barrier, at a resolution between $\sigma^{2}$ and $\sigma^{2}+d \sigma^{2}$, by $d P_{b} / d \sigma^{2}$. Next, we consider the ordinary Press-Schechter barrier, representing the critical overdensity for a region to collapse and form a halo. The differential probability distribution for a random walk to cross the 'collapse barrier', at a resolution between $\sigma^{\prime 2}$ and $\sigma^{\prime 2}+d \sigma^{\prime 2}$, is denoted by $d P_{c} / d \sigma^{\prime 2}$. Similarly, the probability distribution for collapse in a region with large-scale overdensity $\delta_{b}$, on smoothing scale $\sigma^{2}$, is denoted by $d P_{c}\left(\sigma^{\prime 2} \mid \delta_{b}, \sigma^{2}\right) / d \sigma^{\prime 2}$. The total ionized mass in a region of large scale overdensity $\delta_{b}$, at a smoothing scale $\sigma^{2}$, is then given by

$$
\int d M_{h} \zeta M_{h} \frac{d \sigma^{\prime 2}}{d M_{h}} \frac{d P_{c}\left(\sigma^{\prime 2} \mid \delta_{b}, \sigma^{2}\right)}{d \sigma^{\prime 2}} .
$$

Note that the conditional probability distribution in this formula is calculated by considering the fraction of random walks, originating at $\left(\delta_{b}, \sigma^{2}\right)$, that cross the collapse barrier at higher resolution (Lacey \& Cole 1993). The mass calculated using Equation (A1) is precisely the ionized mass in an HII region that crosses the 'bubble barrier' at the point $\left(\delta_{b}, \sigma^{2}\right)$. In order to find the total ionized mass in all HII regions, we merely need to integrate over all such crossings, i.e., we integrate Equation A1 over $\sigma^{2}$ weighted by the probability of crossing the bubble barrier. Symbolically, the total ionized mass in the IGM is then given by

$$
\int d \sigma^{2} \frac{d P_{b}\left(\sigma^{2}\right)}{d \sigma^{2}} \int d M_{h} \zeta M_{h} \frac{d \sigma^{\prime 2}}{d M_{h}} \frac{d P_{c}\left(\sigma^{\prime 2} \mid \delta_{b}, \sigma^{2}\right)}{d \sigma^{\prime 2}}
$$

This is one expression for the total ionized mass in the IGM, obtained by summing the ionized mass in all individual HII regions. Our proof of photon conservation is completed by showing that this 'local' expression matches a separate expression, proportional to the global collapse fraction. The total mass in halos is simply

$$
\int d M_{h} M_{h} \frac{d \sigma^{\prime 2}}{d M_{h}} \frac{d P_{c}\left(\sigma^{\prime 2}\right)}{d \sigma^{\prime 2}}
$$

and the total, photon-conserving, ionized mass is just $\zeta$ times this expression. Now, this expression, proportional to the global collapse fraction follows by considering the crossing distribution of the collapse barrier, irrespective of when each random walk crosses the bubble barrier. This result clearly must match that of Equation A1 since for two random variables, $x$ and $y$ with probability distributions $P(x)$ and $P(y), \int d y P(y) \int d x x P(x \mid y)=\int d x x P(x)$, i.e. in one case we are integrating ('marginalizing') over 'bubble crossings', and in the other case we are not. This proves that the pure FZH04 model conserves photons, and our numerical implementation of the FZH04 model with a sharp $k$-space filter indeed conserves photons.

In practice, however the hybrid scheme of $\$ 3$ smoothes the density field with a top-hat in real space, rather than a sharp $k$-space filter. In this case photon conservation is not guaranteed. Specifically, the expression in Equation (1) of 33 is rigorously equal to the collapse fraction only for sharp $k$-space filtering, and not for real-space smoothing (see also McQuinn et al. 2005). One option would be to simply apply our algorithm with a sharp $k$-space filter, but we find that this produces artificial features in our ionization maps (ringing in configuration space). For this reason, we prefer to apply our algorithm using a top-hat in real space. In practice this leads to photon non-conservation at the $20 \%$ level, with our algorithm systematically under-shooting the expected ionization, $\bar{x}=\zeta \times f_{\text {coll. }}$. To compare with the radiative transfer simulation, we simply boost the ionizing efficiency to make up for this photon loss, matching the (volume-weighted) ionization fraction in the radiative transfer simulation.

Is photon-conservation fulfilled in our improved 'halo-smoothing' scheme? We consider a simple toy problem to illustrate that our improved scheme also does not quite conserve photons. Imagine two equal luminosity sources in a uniform density field. When the ionized regions surrounding these sources begin to overlap, the spherical top-hat criterion can lead to somewhat unphysical features. This is sketched in the left panel of Figure 11. Our algorithm does not allow for flux from one source to expand the HII region surrounding the second source. Instead of both HII spheres (with initial radius $r_{1}$ ) growing further during overlap, a new ionized region arises between them, the overlap of two spheres with radius $r_{2}=2^{1 / 3} r_{1}$. In Figure 11 we plot the ratio of the ionized volume in our scheme, to the expected, photon-conserving ionized volume. The figure clearly illustrates that our scheme generally loses photons as two bubbles 'merge'. The precise level of photon loss in our 'halo-smoothing' scheme will depend on the ionized fraction, the size distribution of the HII regions, the luminosity and bias of the sources interior to merging bubbles, and the rate of merging bubbles. In practice, the level of photon non-conservation in our halo-smoothing scheme is also at the $20 \%$ level. Again our solution is to uniformly boost the ionizing efficiency of our sources to match the (volume-weighted) ionization fraction in the radiative transfer simulation. Ideally, we would only boost the efficiency in recently merged bubbles where we expect photon loss. In practice, any error associated with this approximation appears small, although the higher frequency of bubble mergers at late stages of reionization makes our scheme slightly less reliable in this regime (see Figure 6).

\section{REFERENCES}

Abel, T., Norman, M. L., \& Madau, P. 1998, ApJ, 523, 66, astro-ph/9812151

Abel, T., \& Wandelt, B. D. 2002, MNRAS, 330, L53, astro-ph/0111033
Babich, D., \& Loeb, A. 2005, astro-ph/0509784

Barkana, R., \& Loeb, A. 2000, ApJ, 539, 20, astro-ph/0001326

Barkana, R., \& Loeb, A. 2002, ApJ, 578, 1, astro-ph/0204139

Barkana, R., \& Loeb, A. 2004a, ApJ, 601, 64, astro-ph/0305470 

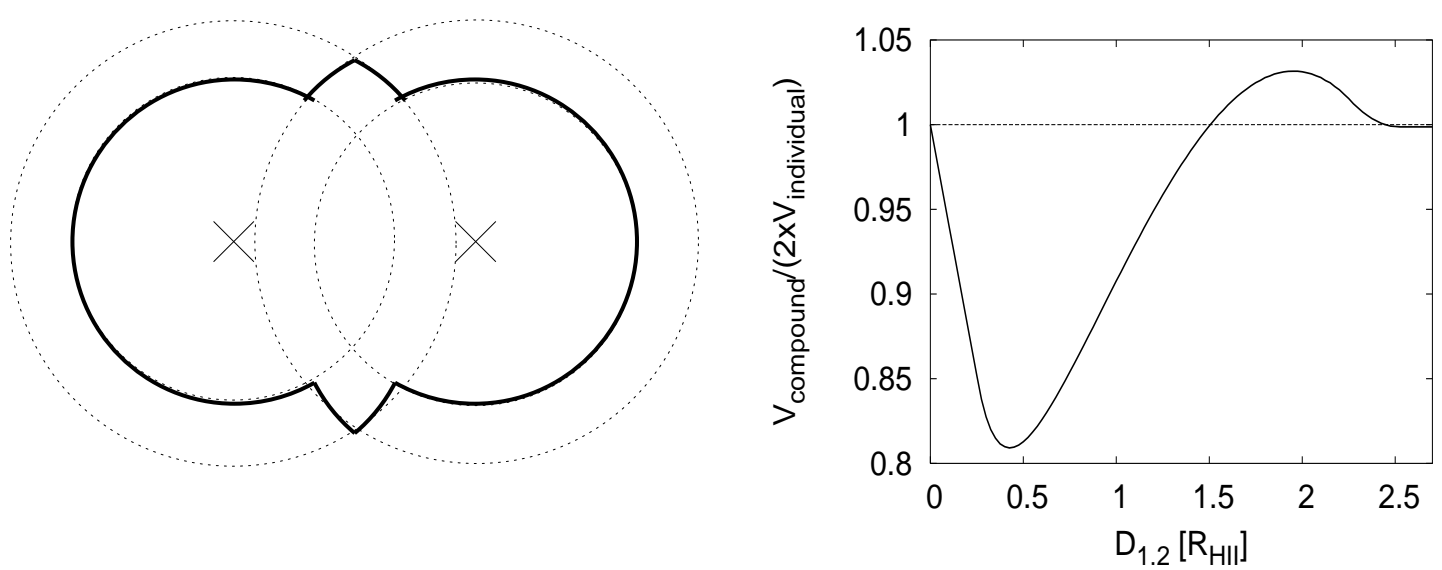

FIG. 11. - An illustration of photon non-conservation in our 'halo-smoothing' simulations. The left panel shows the ionized region from our numerical scheme for the toy problem of two equal-luminosity sources. Our scheme leads to the HII region denoted by the thick solid boundary. In reality, one expects an oblong HII region, as photons from each source stream to the edge of the HII region created by the other source, and expand its volume. For this toy problem, our procedure initially over-estimates the ionized volume by a few percent at moderate source separations, then under-estimates the ionized volume at smaller separations. In the limit of non-overlapping HII regions (very large source separations), and in the limit of very small source separations, we recover the expected ionized volume. This is illustrated in the right panel which shows the fractional photon loss/gain as a function of source separation. The $\mathrm{x}$-axis is the source separation in units of the radius of an individual HII region.

Barkana, R., \& Loeb, A. 2004b, ApJ, 609, 474, astro-ph/0310338 Barkana, R., \& Loeb, A. 2005, ApJ, 624, L65, astro-ph/0409572

Becker, G. D., Sargent, W. L. W., Rauch, M., \& Simcoe, R. A. 2005, ApJ, 640, 69, astro-ph/0511541

Bharadwaj, S., \& Ali, S. S. 2004, MNRAS, 352, 142, astro-ph/0401206

Bond, J. R., Cole, S., Efstathiou, G., \& Kaiser, N. 1991, ApJ, 379,440

Bowman, J. D., Morales, M. F., \& Hewitt, J. N. 2006, ApJ, 638, 20, astro-ph/0507357

Chen, X.-L., \& Miralda-Escude, J. 2004, ApJ, 602, 1, astro-ph/0303395

Ciardi, B., Ferrara, A., \& White, S. D. M. 2003, MNRAS, 344, L7, astro-ph/0302451

Ciardi, B., \& Madau, P. 2003, ApJ, 596, 1, astro-ph/0303249

Cohn, J. D., \& Chang, T.-C. 2006, astro-ph/0603438

Davis, M., Efstathiou, G., Frenk, C. S., \& White, S. D. M. 1985, ApJ, 292, 371

Eisenstein, D. J., \& Hu, W. 1999, ApJ, 511, 5, ADS astro-ph/9710252

Fan, X.-H., et al. 2005, astro-ph/0512082

Furlanetto, S., Zaldarriaga, M., \& Hernquist, L. 2004a, ApJ, 613, 1, astro-ph/0403697 [FZH04]

Furlanetto, S., Zaldarriaga, M., \& Hernquist, L. 2004b, ApJ, 613, 16, astro-ph/0404112

Furlanetto, S. R., McQuinn, M., \& Hernquist, L. 2006a, MNRAS, 365,115 , astro-ph/0507524

Furlanetto, S. R., \& Oh, S. P. 2005, MNRAS, 363, 103, astro-ph/0505065

Furlanetto, S. R., Zaldarriaga, M., \& Hernquist, L. 2006b, MNRAS, 365, 1012, astro-ph/0507266

S. Furlanetto, astro-ph/0604040

Gnedin, N. Y. 2000, ApJ, 542, 535, astro-ph/0002151

Haiman, Z., Abel, T., \& Madau, P. 2000, ApJ, 551, 599, astro-ph/0009125

Haiman, Z., Rees, M. J., \& Loeb, A. 1997, ApJ, 476, 458, astro-ph/9608130

Heitmann, K., Lukic, Z., Habib, S., \& Ricker, P. M. 2006, astro-ph/0601233

Iliev, I. T., et al. 2005, astro-ph/0512187

Kaiser, N. 1987, MNRAS, 227, 1

Kneib, J.-P., Ellis, R. S., Santos, M. R., \& Richard, J. 2004, ApJ, 607, 697, astro-ph/0402319

Kogut, A., et al. 2003, ApJS, 148, 161, astro-ph/0302213

Kohler, K., Gnedin, N. Y., \& Hamilton, A. J. S. 2005 astro-ph/0511627

Kramer, R. H., Haiman, Z. and Oh, S.P. 2006, astro-ph/0604218

Lacey, C., \& Cole, S. 1993, MNRAS, 262, 627, ADS
Lewis, A. 2006, astro-ph/0603753

Lidz, A. Zahn, O., Zaldarriaga, M., McQuinn, M. 2006, in preparation

Loeb, A., Barkana, R., \& Hernquist, L. 2005, ApJ, 620, 553, astro-ph/0403193

Madau, P., Meiksin, A., \& Rees, M. J. 1996, ApJ, 474, 429, astro-ph/9608010

Malhotra, S., \& Rhoads, J. 2005, astro-ph/0511196

McQuinn, M., Furlanetto, S. R., Hernquist, L., Zahn, O., \& Zaldarriaga, M. 2005, ApJ, 630, 643, astro-ph/0504189

McQuinn, M., Zahn, O., Hernquist, L., Zaldarriaga, M., \&

Furlanetto, S. R., astro-ph/0512263

McQuinn, M., et. al, in preparation

A. Mesinger and Z. Haiman, "Evidence for a Boundary of the Cosmological Stromgren Sphere and for

arXiv:astro-ph/0406188.

Mellema, G., Iliev, 1., Alvarez, M., \& Shapiro, P. 2005, astro-ph/0508416

Mellema, G., Iliev, I. T., Pen, U.-L., \& Shapiro, P. R. 2006, astro-ph/0603518

Miralda-Escudé, J., Haehnelt, M., \& Rees, M. J. 2000, ApJ, 530, $1, \mathrm{ADS}$

Oh, S. P. 2002, MNRAS, 336, 1021, astro-ph/0201517

Oh, S. P., \& Haiman, Z. 2003, MNRAS, 346, 456

Page, L., et al. 2006, astro-ph/0603450

Pen, U.-L., Wu, X.-P., \& Peterson, J. 2004, AAS, 205, 131, astro-ph/0404083

Press, W. H., \& Schechter, P. 1974, ApJ, 187, 425

Razoumov, A. O., Norman, M. L., Abel, T., \& Scott, D. 2002, ApJ, 572, 695, ADS

Reed, D., et al. 2005, MNRAS, 357, 82, astro-ph/0312544

Rhoads, J. E., et al. 2003, AJ, 125, 1006, astro-ph/0209544

Santos, M. G., Cooray, A., Haiman, Z., Knox, L., \& Ma, C.-P. 2003, ApJ, 598, 756

U. Seljak, A. Slosar and P. McDonald, arXiv:astro-ph/0604335

Shapiro, P. R., Iliev, I. T., \& Raga, A. C. 2004, MNRAS, 348, 753, astro-ph/0307266

Sheth, R. K., \& Tormen, G. 1999, MNRAS, 308, 119, astro-ph/9901122

Sokasian, A., Abel, T., Hernquist, L., \& Springel, V. 2003, MNRAS, 344, 607, astro-ph/0303098

Sokasian, A., Abel, T., \& Hernquist, L. E. 2001, New Astronomy, $6,359, \mathrm{ADS}$

Sokasian, A., Abel, T., \& Hernquist, L. E. 2002, MNRAS, 332, 601, astro-ph/0112297

Sokasian, A., Yoshida, N., Abel, T., Hernquist, L., \& Springel, V. 2004, MNRAS, 350, 47, astro-ph/0307451

Spergel, D. N., et al. 2006, astro-ph/0603449 
V. Springel and L. Hernquist, 2003, MNRAS, 339, 312, arXiv:astro-ph/0206395

Springel, V. 2005, MNRAS, 364, 1105, astro-ph/0505010

Totani, T., et al. 2005, astro-ph/0512154

Wyithe, J.S.B., Loeb, A., Carilli, C. ApJ, 628, 575, 2005, astro-ph/0411625
Zahn, O., Zaldarriaga, M., Hernquist, L., \& McQuinn, M. 2005, ApJ, 630, 657, astro-ph/0503166

Zahn, O., et. al, in preparation

Zaldarriaga, M., Furlanetto, S. R., \& Hernquist, L. 2004, ApJ, 608,622 , astro-ph/0311514 